\title{
Common-Mode Frequency in Inverter-Penetrated Power Systems: Definition, Analysis, and Quantitative Evaluation
}

This paper was downloaded from TechRxiv (https://www.techrxiv.org).

LICENSE

CC BY-NC-SA 4.0

SUBMISSION DATE / POSTED DATE

03-01-2022 / 07-01-2022

\section{CITATION}

Gao, Huisheng; Xin, Huanhai; Huang, Linbin; Li, Zhiyi; Huang, Wei; Ju, Ping; et al. (2022): Common-Mode Frequency in Inverter-Penetrated Power Systems: Definition, Analysis, and Quantitative Evaluation. TechRxiv. Preprint. https://doi.org/10.36227/techrxiv.17738213.v1

$\mathrm{DOI}$ 


\title{
Common-Mode Frequency in Inverter-Penetrated Power Systems: Definition, Analysis, and Quantitative Evaluation
}

\author{
Huisheng Gao, Huanhai Xin, Linbin Huang, Zhiyi Li, Wei Huang, Ping Ju, and Chen Wu
}

\begin{abstract}
As synchronous generators (SGs) are extensively replaced by inverter-based generators (IBGs), modern power systems are facing complicated frequency stability problems. Conventionally, the frequency nadir and the rate of change of frequency (RoCoF) are the two main factors concerned by power system operators. However, these two factors heavily rely on simulations or experiments, especially in a power system with high-penetration IBGs, which offer limited theoretical insight into how the frequency response characteristics are affected by the devices. This paper aims at filling this gap. Firstly, we derive a formulation of the global frequency for an IBG-penetrated power system, referred to as common-mode frequency (CMF). The derived CMF is demonstrated to be more accurate than existing frequency definitions, e.g., the average system frequency (ASF). Then, a unified transfer function structure (UTFS) is proposed to approximate the frequency responses of different types of devices by focusing on three key parameters, which dramatically reduces the complexity of frequency analysis. On this basis, we introduce two evaluation indices, i.e., frequency drop depth coefficient (FDDC) and frequency drop slope coefficient (FDSC), to theoretically quantify the frequency nadir and the average RoCoF, respectively. Instead of relying on simulations or experiments, our method rigorously links the system's frequency characteristics to the characteristics of heterogeneous devices, which enables an indepth understanding regarding how devices affect the system frequency. Finally, the proposed indices are verified through simulations on a modified IEEE 39-bus test system.
\end{abstract}

Index Terms - Common-mode frequency (CMF), frequency nadir, evaluation of frequency responses, rate of change of frequency (RoCoF), unified transfer function structure (UTFS).

\section{INTRODUCTION}

W ith the ever-increasing integration of renewables, the modern power system is gradually dominated by inverter-based generators (IBGs) [1]. Unlike synchronous generators (SGs), IBGs (e.g., adopting the widely-used gridfollowing control) commonly do not provide frequency support and thus the power system frequency stability is increasingly concerned [2]. For instance, recent blackouts in the United Kingdom and Texas have caused the system frequencies

This work was jointly supported by the 2021 Science and Technology Project of Yunnan Power Grid Company Limited (Research and Application of Online Frequency Strength Assessment and Early Warning Technology for Asynchronous Power Grid with High Penetration of Renewable Generation) and National Natural Science Foundation of China (No. 51922094). dropped to $48.8 \mathrm{~Hz}$ and $59.3 \mathrm{~Hz}$, respectively [3], [4]. It has been widely acknowledged that in-depth analyses and effective evaluations of frequency stability are essential in modern power systems.

Frequency nadir and the rate of change of frequency (RoCoF) are two commonly acknowledged parameters to characterize the frequency stability [5]-[6]. Note that the frequency responses of the different buses in a power network may have different nadirs and RoCoFs during the transients, which makes it complicated to analyze the overall frequency response characteristics. To simplify the analysis, the notion of global frequency (namely, the average of bus frequencies, representing the frequency motion of all the buses as a whole) is usually adopted, which ignores the frequency deviation of each bus from the global frequency.

Existing studies on the evaluation of global frequency are mostly based on the average system frequency (ASF) [7]. However, the theoretical basis of the ASF is not rigorous, and it even cannot be guaranteed that the obtained frequency response pertains to the real global frequency. By assuming that all the generation units are homogeneous, it is pointed out in [8] and [9] that the global frequency is in fact a frequency component decomposed from the bus frequencies, which is identical to the ASF. That is, [8]-[9] have provided some theoretical basis for the ASF. However, the practical settings of modern power systems generally do not meet the assumption of homogeneity, especially when both SGs and IBGs are considered. To this end, [10] proposed a method to obtain the global frequency in a power system with heterogeneous devices. However, this study did not consider the impact of network losses on the system frequency response. In fact, the constant impedance loads can significantly affect the system frequency response [11], which can be viewed as network losses when eliminating the pertinent buses. Hence, the influence of network losses is not negligible, which should be taken into account to obtain the global frequency response.

Currently, there exist many indices to study the global frequency [1], [12]. Among them, the system's inertia is usually

Huisheng Gao, Huanhai Xin, Zhiyi Li and Ping Ju are with the College of Electrical Engineering, Zhejiang University, Hangzhou, China. (Emails: \{gaohuisheng, xinhh, zhiyi, pju\}@zju.edu.cn)

Linbin Huang is with the Department of Information Technology and Electrical Engineering at ETH Zurich, Switzerland. (Email: linhuang@ethz.ch)

Wei Huang and Chen Wu are with the Dispatching Center of Yunnan Power Grid Corporation, Kunming, China. 
considered as an important parameter to evaluate the RoCoF, as the inertia is inversely proportional to the initial value of the RoCoF after disturbances [13]. Hence, the inertia can be used to indicate the frequency change trend in a short period of time and estimate how fast the frequency drops to the nadir, as the dynamics of turbine governors are slow [14]. However, in modern power systems with fast frequency regulations provided by IBGs, different buses have different RoCoF and the RoCoF may quickly decrease from its initial value in an IBG. Therefore, the average RoCoF over a certain period of time is more meaningful. However, the average RoCoF cannot be quantified simply by inertia, and thus needs more investigation.

By comparison, the frequency nadir is highly related to the regulation dynamics of all the generation units in a power system. However, it is in general difficult to use a simple index to quantify the nadir in an IBG-dominated system as the device models are complicated. For simplicity, many works used the reduced models of generation units. In [15], the turbine governors of SGs were simplified as an integrator. However, the obtained frequency response characteristics may not be accurate enough. Ref. [16] proposed to approximately characterize the turbines of all SGs with an identical time constant, and thus, the whole system is reduced to a secondorder system. However, this approach only applies to turbines with a simple structure and cannot handle more complex models. The simplified model of SGs in [17] is more precise and has a wider range of applications, but it cannot deal with power electronic converters. Ref. [18] simplified and aggregated all the generation units as a whole in renewableintegrated power systems, and presented an analytic formula for the frequency response. Ref. [19] mapped a change of the key system variables such as kinetic energy onto the change of frequency to assess the frequency characteristics in power system planning studies. However, [18] and [19] are both based on simulations, which limit the further theoretical analysis. Therefore, there is an urgent need for proper approaches to simplify all types of generation units as well as proper indices to quantify the frequency response characteristics.

To this end, this paper rigorously derives the formula of the global frequency in a power system considering heterogeneous devices and network losses. Moreover, we explicitly show how the frequency responses of different devices can be represented by three key parameters, and how this representation facilitates the frequency evaluation in a system level. Further, we propose two indices to effectively characterize the frequency nadir and the average RoCoF. The main contributions of this paper are summarized below.

1) The derived global frequency, referred to as commonmode frequency (CMF), is not only more theoretically rigorous, but also can obtain more accurate frequency trajectories than the ASF. Moreover, we demonstrate that different generation units and disturbances have different weightings on the global frequency according to their locations, which can be explicitly reflected by the proposed CMF. We will further show that these weightings are related to the network losses.

2) A unified transfer function structure (UTFS) is used to approximately model different types of generation units (including SGs and IBGs), which parameterizes a device's frequency characteristics using only three parameters. Then, the frequency regulation capabilities of heterogeneous devices can be quantified based on the three UTFS parameters. These parameters are insightful to understand the differences of the generation units, e.g., grid-following (GFL) and grid-forming (GFM) dynamics [20].

3) The proposed two indices can explicitly quantify the frequency response characteristics in a convenient way, which is derived based on the proposed UTFS for different devices. Meanwhile, the formulas of the two indices are simple but precisely capture the influence of the regulations of different devices on the system frequency response, and help to guide the improvement of frequency stability.

The rest of this paper is organized as follows: Section II presents the modeling of a representative inverter-penetrated power system, derives the CMF from the bus frequencies based on the spectral decomposition approach, and compares the CMF with several existing frequency analysis methods. Section III simplifies various generation units to the UTFSs. On this basis, the CMF is reduced to second-order (i.e., damped sinusoid). After that, the nadir and average RoCoF can be expressed in closed forms. Moreover, in Section IV, their expressions are further simplified, leading to two quantitative indices, termed frequency drop depth coefficient (FDDC) and frequency drop slope coefficient (FDSC). In Section V, the proposed method and indices are verified via numerical simulations. Section VI discusses the possible solutions to relax the assumptions made in Section II. The conclusions are drawn in Section VII.

Notation: We denote by $\mathbf{0}$ and $\mathbf{1}$ the column vectors or matrices with appropriate dimensions, whose elements are respectively all 0 and 1 . We use $\boldsymbol{I}$ to denote the identity matrix with appropriate dimensions. We use $|V|$ to denote the amplitude of a voltage and $\omega_{0}$ the nominal frequency in $\mathrm{rad} / \mathrm{s}$. Given a set of variables, e.g., $x_{i}(i=1,2, \ldots, n)$, we use $\operatorname{diag}\left\{x_{i}\right\}$ to denote the diagonal matrix whose $i$-th diagonal element is $x_{i}$. We use "e" and " $s$ " respectively denote the natural logarithm and the Laplace variable, and " $s$ " to denote the diagonal matrix $\operatorname{diag}\{s\}$ with appropriate dimensions. For notational simplicity, " $s$ " is omitted if not confusing the understanding.

\section{COMMON-MODE FREQUENCY}

In this section, we describe the model of a power system with heterogeneous generation units, obtain the bus frequency responses and derive the global frequency, i.e., CMF. Further, the CMF is compared with several existing frequency analysis methods.

\section{A. Modeling of an inverter-penetrated power system}

To simplify the analysis, we make the following assumptions, similar to the existing literature, e.g., [8]:

Assumption 1: The voltage amplitudes of generation units are assumed as constants to focus on the relationship between frequency and active power.

Assumption 2: The nonlinearity of the system is ignored, such as the dead zone of governors, under-frequency load 
shedding, and the nonlinearity of power flow equations.

Assumption 3: The models and parameters of the generation units and the topology of the transmission network are known.

Similar to the modeling of conventional multi-machine power systems, an inverter-penetrated power system can also be divided into two parts: the device part (including SGs, IBGs, etc.), and the transmission network part [21]. In the following, we will give a detailed description of the generation units and the network.

a) Generation units: According to Assumptions 1 and 2, the dynamics of a generation unit are represented by the transfer function from frequency input to active power output (FIAPO). In a matrix notation, all the generation devices' FIAPO transfer functions can be expressed as $\boldsymbol{G}(s)=\operatorname{diag}\left\{G_{i}(s)\right\}$. Then, we have

$$
\Delta \boldsymbol{P}(s)=-\boldsymbol{G}(s) \Delta \boldsymbol{\omega}(s)
$$

where $\Delta$ denotes the perturbed value of a variable; $\Delta \boldsymbol{P}$ and $\Delta \boldsymbol{\omega}$ are vectors composed of $\Delta P_{i}$ and $\Delta \omega_{i}$, which are the $i$-th unit's active power output and frequency, respectively $(i=1,2, \ldots, n$, and $n$ is the number of generation units). The sign before $\boldsymbol{G}(s)$ is negative because the devices provide positive active power when the frequency drops.

Without loss of generality, we consider three types of generation units, including SGs, GFL converters, and GFM converters [20]. The detailed models and the FIAPO transfer functions of these devices are given in Appendix-A.

b) Network: The relationship between frequency and active power in the network part can be formulated based on the power flow equation. To be compatible with the generation part, we need to eliminate the passive buses (i.e., except the generation buses) including the network internal buses and the ZIP load buses (i.e., constant impedance, constant current, and constant power loads) [22]. Then, the dynamics of the network can be expressed as

$$
\Delta \boldsymbol{P}(s)=\omega_{0} \boldsymbol{L}_{\mathrm{r}} \boldsymbol{s}^{-1} \Delta \boldsymbol{\omega}(s)
$$

where $\boldsymbol{L}_{\mathrm{r}}$ is the reduced Laplacian matrix. The detailed bus reduction process and the derivation of (2) are given in Appendix-B. Note that the reduction of constant impedance load buses may increase the network resistance greatly.

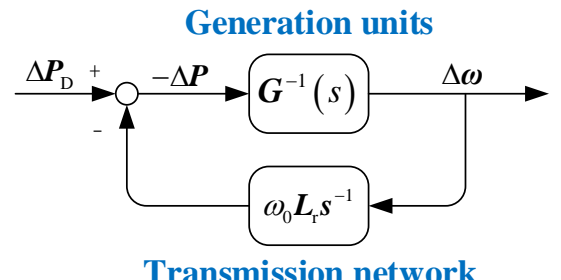

Fig. 1. Diagram of the closed-loop dynamics of an inverter-penetrated power system.

By combining (1) and (2), we obtain the closed-loop dynamics of the power system, as shown in Fig. 1. And it can be deduced from Fig. 1 that the frequency response under power disturbance $\Delta \boldsymbol{P}_{\mathrm{D}}=\left[\Delta P_{\mathrm{D}, 1}, \ldots, \Delta P_{\mathrm{D}, n}\right]^{\mathrm{T}}$ can be expressed as

$$
\Delta \boldsymbol{\omega}(s)=\left(\boldsymbol{G}(s)+\omega_{0} \boldsymbol{L}_{\mathrm{r}} s^{-1}\right)^{-1} \Delta \boldsymbol{P}_{\mathrm{D}}(s)
$$

We note that there are many other types of loads in the power systems, e.g., induction motors. If their voltage dynamics can be ignored (e.g., the voltage fluctuation is relatively small), these types of loads can be generally viewed as special generation units and thus represented by the FIAPO transfer functions. Dealing with loads (generation units) whose voltage dynamics should be taken into account can be included in future works.

\section{B. Derivation of the common-mode frequency}

In (3), the frequency responses can vary from bus to bus. Here we propose an approach to derive the global frequency, i.e., CMF, extracted from the bus frequencies.

In what follows, we firstly introduce some properties of the reduced Laplacian matrix $\boldsymbol{L}_{\mathrm{r}}$ in (3). The matrix $\boldsymbol{L}_{\mathrm{r}}$ has an eigenvalue 0 , with the corresponding right eigenvector being $\mathbf{1}$, as shown in (4) (see Appendix-B and [22] for a detailed discussion). It reflects the rotational invariance of power flow to phase angle, namely, when the phase angles of all buses increase the same value, the power flow does not change. The corresponding left eigenvector of $\boldsymbol{L}_{\mathrm{r}}$ is denoted by $\boldsymbol{w} / n$, where $\boldsymbol{w}=\left[w_{1}, \ldots, w_{n}\right]$ is a row vector.

$$
\boldsymbol{L}_{\mathrm{r}} \mathbf{1}=\mathbf{0}, \quad \boldsymbol{w} \boldsymbol{L}_{\mathrm{r}} / n=\mathbf{0}^{\mathrm{T}}, \boldsymbol{w} \mathbf{1} / n=1
$$

When the network is lossless (i.e., it has no constant impedance loads), $\boldsymbol{L}_{\mathrm{r}}$ is symmetric and $\boldsymbol{w}=\mathbf{1}^{\mathrm{T}}$ (see, e.g., [8][9]). Otherwise, $\boldsymbol{w} \neq \mathbf{1}^{\mathrm{T}}$. An example to show how $\boldsymbol{w}$ changes with different network losses is given in Section V-A. The row vector $\boldsymbol{w}$ will become much different from $\mathbf{1}^{\mathrm{T}}$ if there is a large amount of constant impedance loads.

The above properties of $\boldsymbol{L}_{\mathrm{r}}$ merit attention as the studied global frequency actually characterizes the common component of all the bus frequencies (phase angles). To utilize the property of $\boldsymbol{L}_{\mathrm{r}}$ for the derivation of the CMF, we rewrite (3) as

$$
\boldsymbol{H}(s) \Delta \boldsymbol{\omega}(s)=\boldsymbol{G}^{-1}(s) \Delta \boldsymbol{P}_{\mathrm{D}}(s), \boldsymbol{H}(s):=\boldsymbol{I}+\omega_{0} \boldsymbol{G}^{-1}(s) \boldsymbol{L}_{\mathrm{r}} \boldsymbol{s}^{-1}(5)
$$

By combining (4)-(5), it can be deduced that $\boldsymbol{H}(s)$ has an eigenvalue 1 , and that the corresponding left/right eigenvectors are $\boldsymbol{w} \boldsymbol{G}(s) / \sum_{i}\left(w_{i} G_{i}(s)\right)$ and $\mathbf{1}$. Let $\lambda_{k}(s), \boldsymbol{v}_{k}(s)$ and $\boldsymbol{u}_{k}(s)(k=$ $1,2, \ldots, n)$ respectively denote the eigenvalues and left/right eigenvectors of $\boldsymbol{H}(s)$. The spectral decomposition of $\boldsymbol{H}(s)$ can be expressed as

$$
\begin{gathered}
\boldsymbol{H}(s)=\lambda_{1}(s) \boldsymbol{u}_{1}(s) \boldsymbol{v}_{1}(s)+\sum_{k=2}^{n} \lambda_{k}(s) \boldsymbol{u}_{k}(s) \boldsymbol{v}_{k}(s) \\
\lambda_{1}(s)=1, \boldsymbol{u}_{1}(s)=\mathbf{1}, \boldsymbol{v}_{1}(s)=\frac{\boldsymbol{w} \boldsymbol{G}(s)}{\sum_{i=1}^{n} w_{i} G_{i}(s)}
\end{gathered}
$$

By substituting (6) into (5), $\Delta \omega$ can be decomposed into $n$ components:

$$
\Delta \boldsymbol{\omega}(s)=\sum_{k=1}^{n} \Delta \boldsymbol{\omega}_{k}(s), \Delta \boldsymbol{\omega}_{k}(s)=\frac{\boldsymbol{u}_{k}(s) \boldsymbol{v}_{k}(s) \boldsymbol{G}^{-1}(s)}{\lambda_{k}(s)} \Delta \boldsymbol{P}_{\mathrm{D}}(s)
$$

Among them, $\Delta \omega_{1}(s)$, which corresponds to the eigenvalue $\lambda_{1}(s)$ of $\boldsymbol{H}(s)$ (and eigenvalue 0 of $\boldsymbol{L}_{\mathrm{r}}$ ), can be explicitly expressed as

$$
\Delta \boldsymbol{\omega}_{1}(s)=\mathbf{1} \Delta \omega_{\mathrm{cm}}(s), \text { with } \Delta \omega_{\mathrm{cm}}(s):=\frac{\Delta P_{\mathrm{L}}(s)}{\sum w_{i} G_{i}(s)}
$$

where $\Delta P_{\mathrm{L}}(s)=\boldsymbol{w} \Delta \boldsymbol{P}_{\mathrm{D}}(s)$ is the equivalent disturbance, and $\boldsymbol{w}$ $\left(w_{i}\right)$ is (the element in) the left eigenvector defined in (4). 
Eq. (8) shows that all the elements in $\Delta \boldsymbol{\omega}_{1}$ equal to $\Delta \omega_{\mathrm{cm}}$, which means $\Delta \omega_{1}$ is a global frequency. The essential reason why the elements in $\Delta \omega_{1}$ are identical is that the right eigenvector of $\boldsymbol{L}_{\mathrm{r}}$ corresponding to eigenvalue 0 is $\mathbf{1}$. In this paper, $\Delta \omega_{1}$ (or $\left.\Delta \omega_{\mathrm{cm}}\right)$ is the so-called CMF.

Let $\Delta \boldsymbol{P}_{\mathrm{cm}}=\left[\Delta P_{1, \mathrm{~cm}}, \ldots, \Delta P_{n, \mathrm{~cm}}\right]^{\mathrm{T}}$ denote the generation units' power responses to the $\mathrm{CMF}$ :

$$
\Delta \boldsymbol{P}_{\mathrm{cm}}(s)=-\boldsymbol{G}(s) \Delta \omega_{1}(s), \Delta P_{i . \mathrm{cm}}(s)=-G_{i}(s) \Delta \omega_{\mathrm{cm}}(s)
$$

Note that the weighted sum of $\Delta P_{i, \mathrm{~cm}}$ is equal to the disturbance:

$$
\boldsymbol{w} \Delta \boldsymbol{P}_{\mathrm{cm}}(s)=-\frac{\boldsymbol{w} \boldsymbol{G}(s) \mathbf{1}_{n}}{\sum w_{i} G_{i}(s)} \Delta P_{\mathrm{L}}(s)=-\Delta P_{\mathrm{L}}(s)
$$

Moreover, considering that $\Delta \boldsymbol{\omega}=\sum_{k} \Delta \boldsymbol{\omega}_{k}$ and $\Delta \boldsymbol{P}=-\boldsymbol{G}(s) \Delta \boldsymbol{\omega}$, one can conclude that only part of the device's active power output, i.e., $\Delta P_{i, \mathrm{~cm}}$, participates in response to the disturbance and supports the CMF. In practical power systems, the frequency responses of the buses are dominated by the global component [7]-[14]. Therefore, we can study the system's frequency characteristics and the devices' power supports based on the CMF $\Delta \omega_{\mathrm{cm}}$ and $\Delta \boldsymbol{P}_{\mathrm{cm}}$.

\section{Comparison of CMF with other analysis methods}

For a comprehensive understanding, the CMF is compared with other frequency analysis methods in what follows, such as the ASF [7], the global frequency in [8]-[9], frequency of center of inertia (COI), and frequency divider [23].

a) ASF and global frequency in [8]-[9]: The conventional ASF obtains the global frequency from an equivalent generation unit, which is the sum of all generation units in the power system. The ASF does not model the transmission network and ignores the frequency variations among different buses. In [8]-[9], the generation units and the transmission network are considered, and the global frequency is obtained by decomposing the bus frequencies. Even though the frequency expression in [8]-[9] is the same as the ASF, the decomposition method is more rigorous in theory. However, the derivation in [8]-[9] needs the assumption of homogeneity. It means that $\boldsymbol{G}(s)$ can be expressed as $\boldsymbol{G}(s)=\boldsymbol{F} g_{0}(s)$, where $g_{0}(s)$ is the same part of different devices' transfer functions, and $\boldsymbol{F}=\operatorname{diag}\left\{F_{i}\right\}$ where $F_{i}$ is a scalar. Ref. [8]-[9] decomposes the bus frequencies based on the spectral decomposition of $\boldsymbol{F}^{-1 / 2} \boldsymbol{L}_{\mathrm{r}} \boldsymbol{F}^{-1 / 2}$, thus, the assumption of homogeneity is essential. Moreover, the ASF and [8]-[9] all do not consider the network losses, which will cause certain errors in the obtained frequency trajectory and thus the quantification of frequency response characteristics, especially when there is a large amount of constant impedance loads.

The CMF is also obtained by decomposition, hence it is more rigorous than the ASF. The derivation of CMF is based on the decomposition of the transfer function matrix $\boldsymbol{H}(s)$, which avoids the need for the assumption of homogeneity. Meanwhile, the influence of network losses is taken into account, which is characterized by the weighting coefficients $w_{i}$ in (8). Note that if there are no constant impedance loads and the network losses are negligible, we have $\boldsymbol{w}=\mathbf{1}^{\mathrm{T}}$, and then the CMF will be the same as the ASF. However, if it is not the case, we have $\boldsymbol{w} \neq \mathbf{1}^{\mathrm{T}}$, and then the obtained trajectory of the CMF will be more accurate than the ASF, which will be shown in
Section V-B.

b) COI frequency: The COI frequency is an inertia weighted average of the bus frequencies. The weighting vector is $\boldsymbol{J}_{\mathrm{w}}=$ $\left[J_{1}, \ldots, J_{n}\right]^{\mathrm{T}} / J_{\text {sum }}$, in which $J_{i}$ is the inertia of each generator and $J_{\text {sum }}$ is their sum. To obtain the COI frequency, we should get all the buses' frequency responses, which means COI frequency relies on measurements or simulations. Namely, the COI frequency may not be able to provide much information.

The CMF can also be obtained by weighting the bus frequencies with the weighting vector $\boldsymbol{v}_{1}(s)$ (in (6)), which can be proved based on the orthogonality of eigenvectors. In particular, when devices are homogeneous $\left(G_{i}(s) / G_{j}(s)=J_{i} / J_{j}\right)$ and $\boldsymbol{w}=\mathbf{1}^{\mathrm{T}}$, the weighting vector $\boldsymbol{v}_{1}(s)$ of the CMF will be the same as $\boldsymbol{J}_{\mathrm{w}}$ of the COI frequency. In Section V-B, we will show that when the aforementioned conditions are not met, using $\boldsymbol{v}_{1}(s)$ as the weighting vector can obtain a "smoother" frequency trajectory than using $\boldsymbol{J}_{\mathrm{w}}$.

c) Frequency Divider: The modeling of the transmission network in the derivation of frequency divider and in this paper might be considered as similar in some aspects. However, the key point of the frequency divider is to reveal the relationship between the frequency of the generators and the network buses. It is pointed out that the frequency of a network bus is a linear combination of the generator frequencies, which changes along with the transmission lines. In this paper, we focus on obtaining the global frequency based on the frequencies of all the devices, where the passive network buses are eliminated.

The derivation in this paper can be combined with the frequency divider to illustrate that the CMF is identical in not only the device buses, but also the other reduced buses, which further demonstrates that CMF is indeed the global frequency.

According to the above discussions, it can be concluded that the CMF is more theoretically rigorous than the ASF, and the frequency response characteristics obtained by the CMF are more accurate than the ASF and the global frequency in [8]-[9], especially when there is a huge amount of constant impedance loads in the power system. Moreover, the weighting vector $\boldsymbol{v}_{1}(s)$ of the CMF may be a better choice than the inertia vector $\boldsymbol{J}_{\mathrm{w}}$ of the $\mathrm{COI}$ frequency for an average system frequency.

One possible shortcoming of the $\mathrm{CMF}$ is that it requires more information for calculating the weighting vector $\boldsymbol{w}$, i.e., network topology, steady-state voltage amplitudes and phase angles. These can be obtained in the power flow analysis for a given power system with predetermined operating conditions. Aggregating all the generation units certainly helps to simplify the calculation of $\boldsymbol{w}$, e.g., replacing a solar station with one equivalent device, whose FIAPO transfer function is the sum of all the inverters inside. Such aggregation is valid if the terminal voltage of the station is regulated to be approximately constant and the network losses inside the station are negligible. In this condition, the active power output of the equivalent device will be the same as the original station, as the CMF is identical anywhere in the power systems. In fact, aggregating multiple units can be viewed as ignoring the transmission lines inside the station and the coupling among all the units inside. 


\section{Model Simplification OF Generation UnITS For} ANALYZING FREQUENCY RESPONSE CHARACTERISTICS

The CMF in (8) is much simpler compared with the bus frequencies in (3). However, the frequency response characteristics are still difficult to be analyzed because the order of the CMF is generally high in practice, considering the huge number of generation units and their complex dynamics. In this section, a unified transfer function structure (UTFS) is proposed to approximate the complex dynamics of different types of devices and then analytically describe the frequency response.

\section{A. Unified transfer function structure}

As shown in (8), the transfer functions of the devices are added up. If the transfer function structures of the various devices are different, the overall order of the system will be very high. For example, considering $n$ devices with the transfer functions respectively being $-K_{i} /\left(1+T_{i} s\right)$. If $T_{i} \neq T_{j}$, then the order of the whole system is $n$. In contrast, if all $T_{i}$ are the same, the whole system is only of first-order. Hence, to keep the order of the whole system low, the simplified models of all the generation units need to share the same structure. Here, the UTFS of the various units is given as

$$
G_{\mathrm{u}}(s)=J_{\mathrm{u}} s+D_{\mathrm{u}}+1 /\left(K_{\mathrm{u}} s\right)
$$

where $J_{\mathrm{u}}, D_{\mathrm{u}}$, and $K_{\mathrm{u}}$ are the effective inertia, effective damping, and effective regulation coefficient, respectively. Obviously, when summing up multiple $G_{\mathrm{u}}(\mathrm{s})$ with different parameters, the resulting transfer function still preserves the same form as (11) and we simply need to add up the corresponding parameters.

In fact, many other transfer function structures can be adopted, e.g., replacing the integral term in (11) with a firstorder low-pass filter while the proportional and derivative terms remain the same (e.g., the model in [16]). We choose the structure in (11) for two reasons. On the one hand, based on this UTFS, the approximated frequency response is a damped sinusoid, which is very close to the CMF in the period from the initial moment to the frequency nadir, as shown in Fig. 2. Thus, the frequency nadir and the average RoCoF can be precisely obtained. On the other hand, this structure tends be the simplest one for studying the frequency response characteristics, as it effectively preserves a low-order model, as discussed above.

When other frequency characteristics with a larger time scale are of interest, e.g., the quasi-steady frequency deviation, the UTFS in (11) may not be a suitable choice. In this case, the aforementioned structure with a low-pass filter could be a better choice, which can be studied in future works.

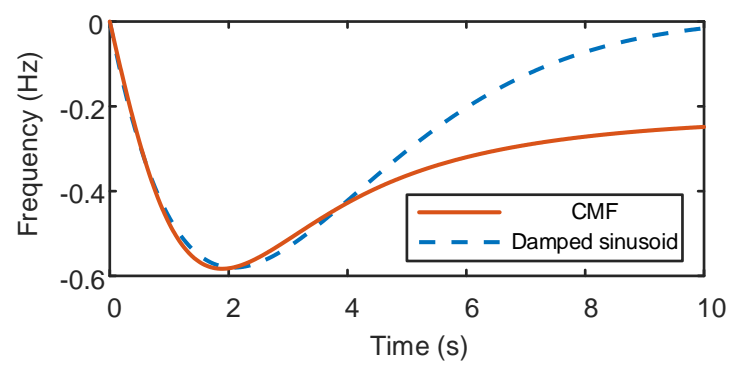

Fig. 2. The trajectory of the CMF and its approximation.

\section{B. Parameter settings of UTFS}

Modeling different devices using UTFS can be interpreted as a parameter optimization problem, i.e., how to choose appropriate $J_{\mathrm{u}, i}, D_{\mathrm{u}, i}$, and $K_{\mathrm{u}, i}$ such that $G_{\mathrm{u}, i}(\mathrm{~s})$ has a similar response to $G_{i}(\mathrm{~s})$, where $G_{\mathrm{u}, i}(\mathrm{~s})$ is the UTFS of the $i$-th device and $J_{\mathrm{u}, i}, D_{\mathrm{u}, i}$, and $K_{\mathrm{u}, i}$ are its parameters. Considering that the characteristics of the CMF are of interest, we only need to focus on the responses of $G_{i}(\mathrm{~s})$ and $G_{\mathrm{u}, i}(\mathrm{~s})$ taking the $\mathrm{CMF}$ as an input. That is, the objective is to approximate $\Delta P_{i, \mathrm{~cm}}$ in (9) by $\Delta P_{\mathrm{u}, i, \mathrm{~cm}}$, with $\Delta P_{\mathrm{u}, i, \mathrm{~cm}}$ being

$$
\Delta P_{\mathrm{u}, i . \mathrm{cm}}(s)=-G_{\mathrm{u}, i}(s) \Delta \omega_{\mathrm{cm}}(s) \quad i=1,2, \ldots n
$$

Moreover, as shown in Fig. 2, the damped sinusoid is similar to the CMF only at the beginning of the frequency event. Consequently, the optimization objective needs to focus on this period. Based on the above analysis, the parameter optimization problem of the UTFS in each generation unit is described as:

$$
\begin{array}{cc}
\min _{J_{\mathrm{u}, i}, D_{\mathrm{u}, i}, K_{\mathrm{u}, i}} & \int_{t=t_{0}}^{t_{\mathrm{f}}}\left(\Delta P_{\mathrm{u}, i, \mathrm{~cm}}(t)-\Delta P_{i . \mathrm{cm}}(t)\right)^{2} \mathrm{dt} \\
\text { s.t. } & (8),(9),(11),(12)
\end{array}
$$

where $t_{0}$ is the time when a disturbance begins and $t_{\mathrm{f}}$ can generally be chosen as $1.5 t_{\text {nadir }}$, with $t_{\text {nadir }}$ the time of frequency nadir. If the system frequency response is close to the damped sinusoid for a longer period of time (e.g., Cases 1, and 6 in Fig. 9), $t_{\mathrm{f}}$ can be extended accordingly.

As shown in (11) and (12), $\Delta P_{\mathrm{u}, i, \mathrm{~cm}}$ consists of three terms (i.e., $s \Delta \omega_{\mathrm{cm}}, \Delta \omega_{\mathrm{cm}}$ and $\left.\Delta \omega_{\mathrm{cm}} / s\right)$. Therefore, the optimization problem can be described as approximating $\Delta P_{i, \mathrm{~cm}}$ by the linear combination of these three terms, with the optimal coefficients being $J_{\mathrm{u}, i}, D_{\mathrm{u}, i}$ and $1 / K_{\mathrm{u}, i}$, respectively. Therefore, the problem can be solved by least-square methods. One difficulty in solving the problem is that $\Delta P_{\mathrm{u}, i, \mathrm{~cm}}(t)$ and $\Delta P_{i, \mathrm{~cm}}(t)$ are calculated by taking the CMF as the input, and the computational burden may become unacceptable when the order of CMF is very high. We proposed an iterative method to solve this problem, which will be further discussed in the next subsection.

Note that the assumption of linearity of the device is not essential in the above optimization problem, because all we need are the power responses $\left(\Delta P_{\mathrm{u}, i, \mathrm{~cm}}\right.$ and $\left.\Delta P_{i, \mathrm{~cm}}\right)$ of the device, which can be obtained even the device model is nonlinear. However, if it is nonlinear, the UTFS parameters will change with the amplitude of the CMF, (e.g., if the frequency deviation is smaller than the dead zone, the governor will not act), which is different from the linear case.

It is also worth mentioning that the above approximation can be described as quantifying the nonstandard frequency regulations by the three selected standard forms. For example, consider a generation unit that provides virtual inertia with a transfer function $-J_{\mathrm{V}} s /\left(1+T_{f} s\right)$. Apparently, the virtual inertia $J_{\mathrm{V}}$ cannot properly characterize the regulation capability of the device, especially when $T_{f}$ is large. In contrast, by converting the device into the UTFS, its dynamics are then described by not only the inertia but also the damping and the integral of the frequency. Hence, the parameter $J_{\mathrm{u}}$ of UTFS will correspond to only a part of the device's active power response which is close to the response of the "true" inertia. Thus, the parameter $J_{\mathrm{u}}$ can 
characterize the device's inertia property more effectively than the parameter $J_{\mathrm{V}}$. That is why $J_{\mathrm{u}}$ is called the "effective" inertia, so are $D_{\mathrm{u}}$, and $K_{\mathrm{u}}$.

Note that the chosen standard regulation forms are related to the system's frequency response. Consequently, the UTFS parameters of a generation unit may change when the system's operating conditions vary. One exception is when the device's dynamics are strictly in consistency with the UTFS, e.g., the rotor inertia. Besides, the UTFS parameters will still be valid if the system CMF does not change significantly, which is the basis of parametric variation analysis.

\section{Approximated common-mode frequency using UTFS}

By replacing the $G_{i}(s)$ in (8) with UTFS $G_{\mathrm{u}, i}(\mathrm{~s})$, the CMF can be approximately expressed as

$$
\Delta \omega_{\mathrm{cm}}^{\prime}(s)=\frac{1}{J_{\mathrm{us}} s+D_{\mathrm{us}}+1 /\left(K_{\mathrm{us}} s\right)} \Delta P_{\mathrm{L}}(s)
$$

where $J_{\text {us }}, D_{\text {us }}$, and $1 / K_{\text {us }}$ are the UTFS parameters of the whole system, which can be obtained by directly summing up the UTFS parameters of all the devices

$$
J_{\mathrm{us}}=\sum_{i=1}^{n} w_{i} J_{\mathrm{u}, i}, D_{\mathrm{us}}=\sum_{i=1}^{n} w_{i} D_{\mathrm{u}, i}, \frac{1}{K_{\mathrm{us}}}=\sum_{i=1}^{n} \frac{w_{i}}{K_{\mathrm{u}, i}}
$$

We note that although each device is simplified to UTFS independently, the weighted sums of all the devices' UTFS parameters (i.e., $J_{\mathrm{us}}, D_{\mathrm{us}}$, and $1 / K_{\mathrm{us}}$ in (15)) will be the optimal UTFS parameters of the entire system. This is because the used least-square method is linear and the transfer function of the system (i.e., $\left.\sum_{i}\left(w_{i} G_{i}(s)\right)\right)$ is the linear combination of each device $G_{i}(s)$.

Compared with the original CMF in (8), the approximated $\mathrm{CMF}$ in (14) is only of second-order and much easier to be analyzed, which demonstrates the advantages of the UTFS.

Meanwhile, the approximated CMF is also more suitable to be substituted to (13) for solving the optimization problem. To utilize the simplicity of the approximated CMF, the following iterative method is needed:

1) Estimate the initial value of all the generation units' UTFS parameters;

2) Based on all the generation units' UTFSs, calculate the approximated CMF in (14).

3) Solve the optimization problem (13) based on the approximate CMF and update each unit's UTFS parameters;

4) Iterate steps $2 \sim 3$ until the parameters converge.

\section{QUANTIFICATION AND EVALUATION OF FREQUENCY RESPONSE CHARACTERISTICS}

In this section, we propose two indices to quantitatively evaluate the frequency nadir and the average RoCoF based on the UTFS and CMF. These two indices intuitively reveal how the devices in the power grid affect the system frequency.

\section{A. Quantification of frequency response characteristics}

Considering a power step disturbance, $\Delta P_{\mathrm{L}}=\boldsymbol{w} \Delta \boldsymbol{P}_{\mathrm{D}}=-P_{0} / \mathrm{s}$ ( $P_{0}$ is the equivalent step size), the approximated CMF in (14) can be rewritten as

$$
\Delta \omega_{\mathrm{cm}}^{\prime}(s)=-\frac{P_{0}}{J_{\mathrm{us}} s^{2}+D_{\mathrm{us}} s+\frac{1}{K_{\mathrm{us}}}}
$$

The nadir and average RoCoF of $\Delta \omega_{\mathrm{cm}}^{\prime}$ in (16) can be analytically obtained. However, their expressions are still complicated and may not be intuitive. Thus, we give the following proposition.

Proposition 1. Let $\zeta=D_{\text {us }} /\left(2 \sqrt{J_{\text {us }} / K_{\text {us }}}\right), \quad \omega_{\mathrm{n}}=1 / \sqrt{J_{\text {us }} K_{\text {us }}}$, $\omega_{\mathrm{d}}=\omega_{\mathrm{n}} \sqrt{1-\zeta^{2}}$, and $\sigma=-\zeta \omega_{\mathrm{n}}$ respectively denote the damping ratio, undamped oscillation frequency, damped oscillation frequency, and attenuation coefficient of the second-order system in (16). When $0 \leq \zeta \leq 2.5$, the following two conclusions hold.

a) The frequency nadir $\Delta \omega_{\text {nadir }}$ of $\Delta \omega_{\mathrm{cm}}^{\prime}$ and the corresponding time $t_{\text {nadir }}$ can be expressed as

$$
\left\{\begin{array}{l}
\Delta \omega_{\text {nadir }}=-P_{0} \sqrt{\frac{K_{\text {us }}}{J_{\text {us }}}} \mathrm{e}^{\frac{\sigma}{\omega_{\mathrm{d}}} \arctan \left(-\frac{\omega_{\mathrm{d}}}{\sigma}\right)} \\
t_{\text {nadir }}=\arctan \left(-\frac{\omega_{\mathrm{d}}}{\sigma}\right) \cdot \frac{1}{\omega_{\mathrm{d}}}
\end{array}\right.
$$

Moreover, the frequency nadir $\Delta \omega_{\text {nadir }}$ can be estimated by an empirical formula:

$$
\Delta \omega_{\text {nadir }}^{\prime}=-\frac{P_{0}}{\frac{\mathrm{e}-1}{2} D_{\mathrm{us}}+\sqrt{\frac{J_{\mathrm{us}}}{K_{\mathrm{us}}}}}
$$

The relative error between $\Delta \omega_{\text {nadir }}$ and $\Delta \omega^{\prime}$ nadir is less than $5 \%$.

b) Consider $t_{1}=t_{\text {nadir }} / n_{\mathrm{t}}$, in which $n_{\mathrm{t}}$ is a positive integer. Then, the average RoCoF within $0 \sim t_{1}$, i.e., $\Delta \dot{\omega}_{01 t_{1}}=\Delta \omega_{\mathrm{cm}}^{\prime}\left(t_{1}\right) / t_{1}$ can be estimated by the following empirical formula:

$$
\Delta \dot{\omega}_{01 t_{1}}^{\prime}=-\frac{P_{0}}{J_{\text {us }}[(\mathrm{e}-1) \zeta+1]^{\frac{1}{n_{\mathrm{t}}}}}
$$

When $n_{\mathrm{t}}=3$, the relative error between $\Delta \dot{\omega}_{01 t_{1}}$ and $\Delta \dot{\omega}_{01}^{\prime} t_{1}$ is less than $5 \%$.

Proof: see Appendix-C.

The assumption $0 \leq \zeta \leq 2.5$ indicates that both $J_{\text {us }}$ and $1 / K_{\text {us }}$ are not significantly small compared with $D_{\text {us }}$, which can be satisfied as enough inertia $\left(J_{\text {us }}\right)$ is generally required by the grid. Besides, the dynamics of the turbine governor are slow, which will be mainly approximated as the integral of frequency $\left(1 / K_{\mathrm{us}}\right)$ in the UTFS, it is then inferred that the damping ratio is typically less than 2.5 in power systems with a certain proportion of SGs. Thus, we can quantify the nadir and average RoCoF based on Proposition 1. How to deal with power systems with $\zeta>2.5$ (e.g., 100\% IBGs) will be our future works.

a) Frequency nadir

Based on Proposition 1-a, the FDDC $\alpha$ is proposed to quantify the frequency nadir, which is the inverse of the nadir under the unit power step disturbance as 


$$
\alpha=\frac{\mathrm{e}-1}{2} D_{\mathrm{us}}+\sqrt{\frac{J_{\mathrm{us}}}{K_{\mathrm{us}}}}
$$

\section{b) Average RoCoF}

Based on Proposition 1-b, the FDSC $J_{\mathrm{z}}$ is proposed to quantify the average RoCoF, which is the inverse of the average RoCoF within $0 \sim t_{1}\left(t_{1}=t_{\text {nadir }} / 3\right)$ under the unit power step disturbance as

$$
J_{\mathrm{z}}=J_{\text {us }} \sqrt[3]{(\mathrm{e}-1) \zeta+1}
$$

Note that the average RoCoF may be more meaningful than the initial one in inverter-penetrated power systems, which will be discussed in Section V-C in detail.

The two indices are independent of the disturbance, namely, they reflect the inherent power system properties. Besides, their expressions link the system's frequency characteristics to the characteristics of devices and help understand the influences of the three frequency regulation forms (i.e., the regulation power proportional to the frequency itself, the differential, and the integral) on the system frequency response. To be more specific:

1) According to (20), increasing the inertia $J_{\mathrm{us}}$, damping $D_{\mathrm{us}}$, or $1 / K_{\text {us }}$ can all increase the nadir. Since the inertia is squared and is multiplied by $1 / K_{\mathrm{us}}$, increasing $D_{\mathrm{us}}$ is prone to be more effective than increasing $J_{\text {us }}$ under the scenario where the power systems already have much inertia or a small $1 / K_{\text {us }}$.

2) According to (21), the FDSC is affected by both the inertia and the damping ratio. That is, we can slow down the frequency change rate by increasing either the inertia or the damping in a device level.

It is worth mentioning that the above parametric variation analysis is based on the UTFS parameters, and compatible with the traditional parameters such as SG inertia, virtual inertia, and damping of the GFM converters, as these parameters can be converted to the UTFS parameters directly. However, the relationship between the parameters such as time constants of a turbine-governor and the UTFS may be difficult to be analytically established, which needs further studies.

\section{B. Evaluation of frequency response characteristics}

The critical values of the two indices can be calculated by:

$$
\alpha_{\mathrm{c}}=\left|P_{0}\right| /\left|\Delta \omega_{\text {nadir_max }}\right|, \quad J_{\mathrm{zc}}=\left|P_{0}\right| /\left|\Delta \dot{\omega}_{01 \text { t1_max }}\right|
$$

where $\alpha_{\mathrm{c}}$ and $J_{\mathrm{zc}}$ are the critical values of the indices; $\left|\Delta \omega_{\text {nadir_max }}\right|$ and $\left|\Delta \dot{\omega}_{01 \text { t1_max }}\right|$ are the maximal allowed amplitude of the frequency nadir and the average RoCoF, respectively.

Then, the frequency stability margins can be defined as:

$$
m_{\text {nadir }}=\frac{\alpha-\alpha_{\mathrm{c}}}{\alpha_{\mathrm{c}}}, \quad m_{\mathrm{ARoCoF}}=\frac{J_{\mathrm{z}}-J_{\mathrm{zc}}}{J_{\mathrm{zc}}}
$$

When the two indices are larger than their critical values, the margins $m_{\text {nadir }}$ and $m_{\mathrm{ARoCoF}}$ are both positive, and the two frequency factors meet the requirements. The larger the indices, the larger the margins. On the contrary, if $m_{\text {nadir }}$ or $m_{\mathrm{ARoCoF}}$ is negative, the system's frequency regulation capability needs to be enhanced accordingly.

The quantitative evaluation process of frequency response characteristics can be summarized as follows, which is also shown in Fig. 3.

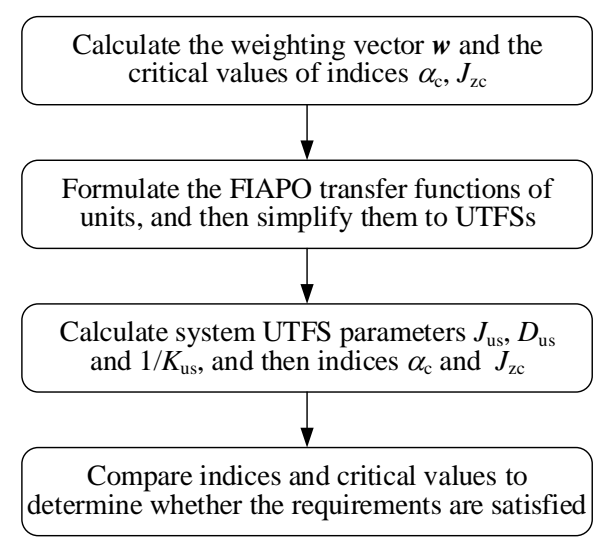

Fig. 3. Flow chart of the frequency evaluation.

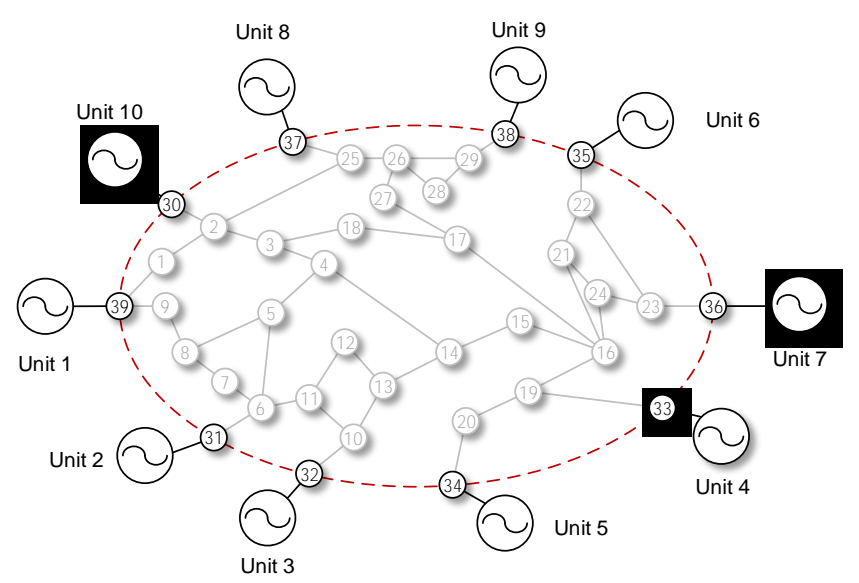

Fig. 4. The 39-bus test system.

1) Form the matrix $\boldsymbol{L}_{\mathrm{r}}$ according to the power network (see Appendix-B) and calculate the weighting vector $\boldsymbol{w}$. Determine the disturbance (size and location) and the allowed amplitude of nadir, average RoCoF. Then calculate equivalent disturbance size $P_{0}$ and critical values of indices $\alpha_{\mathrm{c}}$ and $J_{\mathrm{zc}}$ according to (22).

2) Formulate the FIAPO transfer functions of the generation units based on their models, and then simplify them into the UTFSs using the method in Section III-B, C.

3) Sum up the UTFS parameters of all the units weighted by $w_{i}$ to get the system's parameters, i.e., $J_{\mathrm{us}}, D_{\mathrm{us}}$ and $1 / K_{\mathrm{us}}$. Then calculate FDDC and FDSC based on (20) and (21).

4) Compare the indices with the corresponding critical values (23) to determine whether the system's frequency nadir and average RoCoF meet the requirements.

\section{CASE Studies}

In this section, the effectiveness of the CMF, UTFS and the proposed indices are verified via numerical simulations on a modified IEEE 39-bus system.

\section{A. Test system descriptions}

The 39-bus system used in this section is modified based on the standard New England IEEE 39-bus system [24], as shown in Fig. 4. The network topology of the test system is the same as the standard system. Three types of generation units are used in the study, including SGs, GFL and GFM converters. Their models and FIAPO transfer functions are presented in 
Appendix-A. To facilitate the comparison of different types of generation units, all units have the same capacity of 1000 MVA, and the main parameters of the same type of units are identical, as shown in TABLE I, unless otherwise specified. Note that all units have the same inertia or virtual inertia $\left(J_{\mathrm{SG}}, J_{\mathrm{GFL}}, J_{\mathrm{GFM}}\right)$ and the same primary frequency control (PFC) coefficient $\left(K_{\mathrm{SG}}\right.$, $\left.D_{\mathrm{GFL}}, D_{\mathrm{GFM}}\right)$. The base value of the power is chosen as 1000 MVA.

To show the advantages of CMF over ASF, we consider a system which has a large amount of constant impedance loads, located at bus 15, 16, 18, 21, 24, 26, 27 and 28, consuming about 8 p.u. active power and 0.8 p.u. reactive power in total. The active power setpoint of each device is $0.8 \mathrm{p}$.u, except that the setpoint of G10 is adjusted to maintain system frequency at a nominal value $60 \mathrm{~Hz}$ in the initial steady-state. In all the following cases, the power flows in initial steady-state are set to be the same. That is, the weighting vector $w$ is almost identical in all cases, as shown in TABLE II. The largest and smallest weighting coefficients are respectively $w_{8}=1.0783$ and $w_{9}=0.8138$, which means the impact of generation unit or disturbance located in bus $37\left(w_{8}\right)$ will be $32.5 \%$ larger than that in bus $38\left(w_{9}\right)$.

TABLE I. Main Parameters of Generation Units.

\begin{tabular}{lccccc}
\hline & \multicolumn{5}{c}{ Main Parameters } \\
\cline { 2 - 6 } Unit & $\begin{array}{c}\text { Inertia/ Virtual } \\
\text { Inertia (p.u.) }\end{array}$ & $\begin{array}{c}\text { PFC Coefficient } \\
\text { (p.u.) }\end{array}$ & $\begin{array}{c}\text { Control Gain } \\
\text { (p.u.) }\end{array}$ & Time Constiant (s) \\
\hline \multirow{4}{*}{ SG } & \multirow{3}{*}{$J_{\mathrm{SG}}=8$} & $D_{\mathrm{SG}}=0$ & $K_{1}=0.22$ & $T_{1}=0.2$ & $T_{2}=0.22$ \\
& & $K_{\mathrm{SG}}=20$ & $T_{3}=0.1$ & $T_{4}=0.25$ \\
& & & $K_{7}=0.26$ & $T_{7}=0.4$ & $T_{6}=4$ \\
\hline \multirow{2}{*}{ GFL } & \multirow{2}{*}{$J_{\mathrm{GFL}}=8$} & \multirow{2}{*}{$D_{\mathrm{GFL}}=20$} & $K_{\mathrm{P}}=0.16$ & $T_{\mathrm{F}}=0.01$ & $T_{\mathrm{O}}=0.1$ \\
& & & $K_{\mathrm{I}}=3.71$ & $T_{\mathrm{D}}=0.01$ & \\
\hline GFM & $J_{\mathrm{GFM}}=8$ & $D_{\mathrm{GFM}}=20$ & - & - & - \\
\hline
\end{tabular}

TABLE II. Weighting Coefficients.

\begin{tabular}{ccccc}
\hline$w_{1}$ & $w_{2}$ & $w_{3}$ & $w_{4}$ & $w_{5}$ \\
1.0038 & 1.0551 & 1.0615 & 1.0307 & 1.0208 \\
\hline$w_{6}$ & $w_{7}$ & $w_{8}$ & $w_{9}$ & $w_{10}$ \\
0.9640 & 0.9635 & 1.0783 & 0.8138 & 1.0085 \\
\hline
\end{tabular}

TABLE III. Cases Description.

\begin{tabular}{ccc}
\hline Case & Generation Units & Disturbances \\
\hline 1 & G2, G4: GFL; G6, G8: GFM; The rest: SG. & G8, 0.3p.u. \\
\hline 2 & All units: SG. & 2-a: G8, 0.3p.u. \\
2-b: G9, 0.3p.u.
\end{tabular}

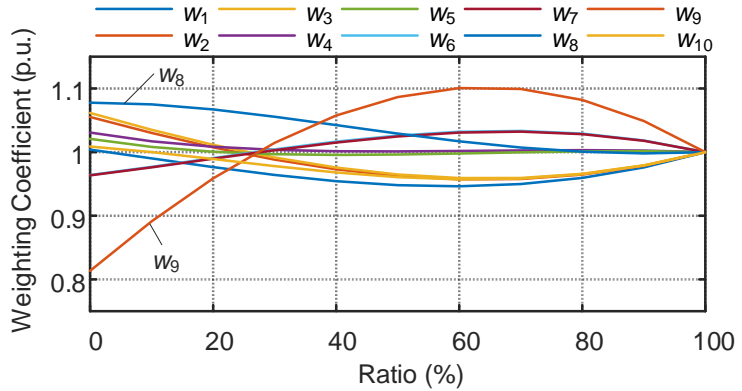

Fig. 5 Changes of the weighting coefficients when the constant impedance loads and network losses reduce, where " $100 \%$ " means there are no constant impedance loads and network losses.

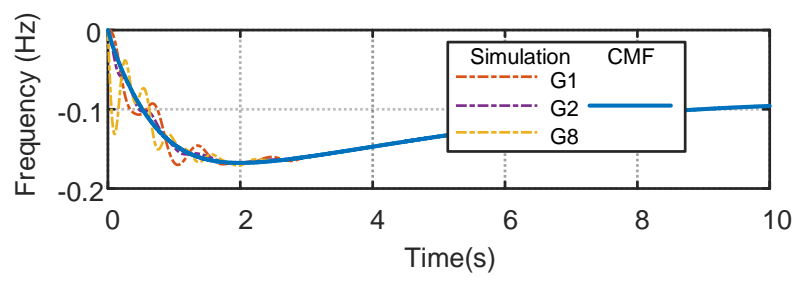

(a)

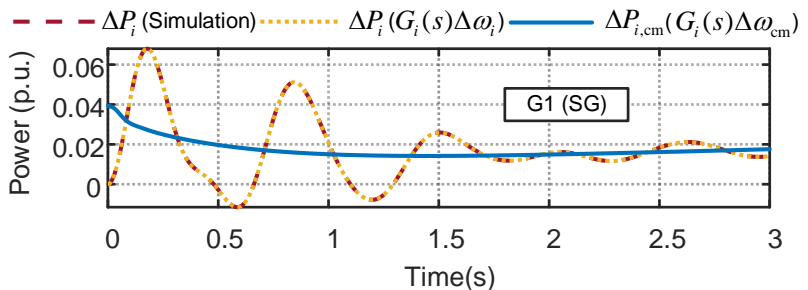

(b)

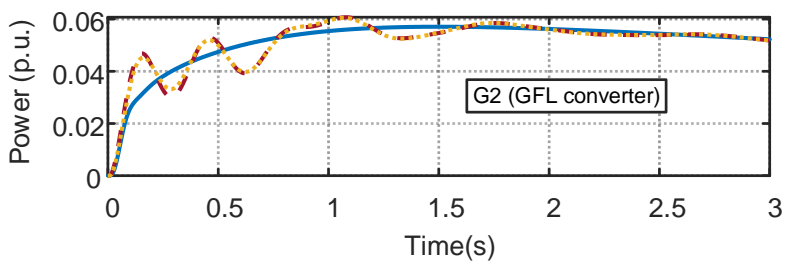

(c)

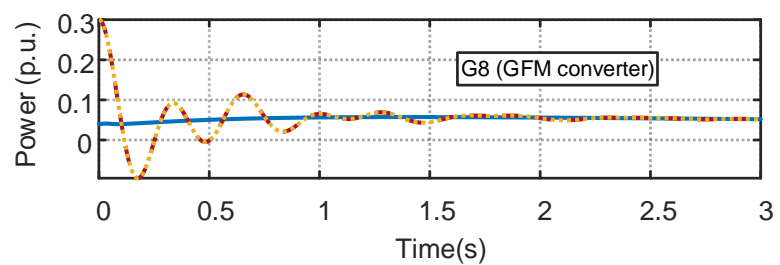

(d)

Fig. 6. Time-domain simulation and theoretical results in Case 1. The CMF and the frequency responses of G1, G2 and G8 (a). The actual active power responses and power responses under CMF of G1 (b), G2 (c) and G8 (d).

Fig. 5 demonstrates how the weighting vector changes with less constant impedance loads and network losses (the power flow is recalculated with the changes). As expected, when there are no constant impedance loads and network losses, the weighting coefficients are all 1 . They do not change with loads linearly due to the nonlinearity of the power flow.

We consider 7 cases with different settings in the generation units and the disturbances, as shown in TABLE III. In the following, the time-domain simulation is conducted in the MATLAB/Simulink, running on a PC with $2.5 \mathrm{GHz} \mathrm{CPU}$ and 16 GB RAM. 

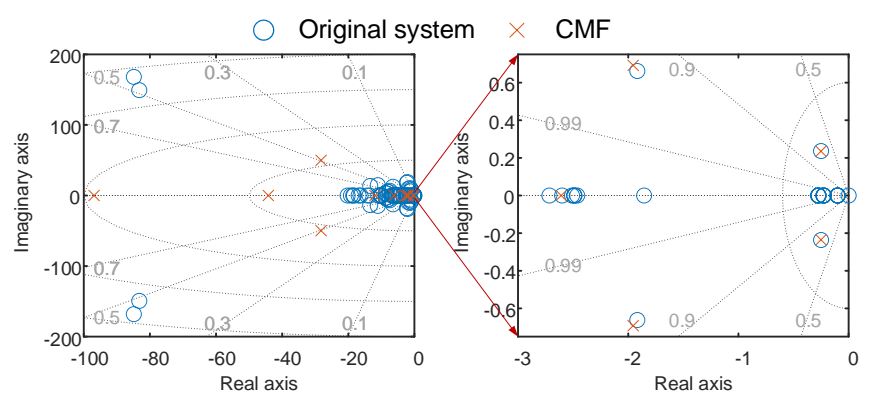

Fig. 7. Eigenvalues of the original system and CMF in Case 1.

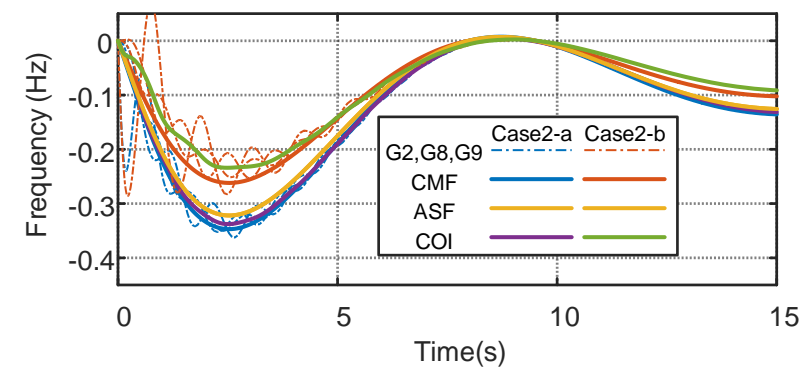

(a)

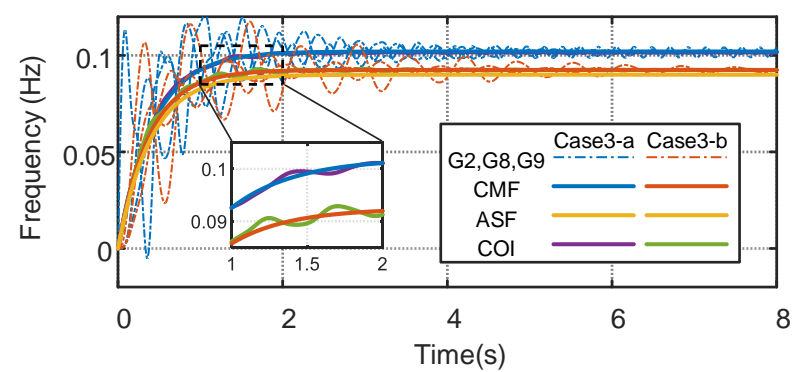

(b)

Fig. 8. Trajectories of CMF, ASF, COI frequency and time-domain simulation results in Case 2 (a) and Case 3 (b)

\section{B. Effectiveness of the CMF}

The validity of the CMF is verified based on Cases 1,2 and 3 , in which Case 1 focuses on the representativeness of the CMF to all bus frequencies and the correctness of the derived FIAPO transfer functions. Cases 2 and 3 are used to compare the CMF with other methods, such as the ASF and COI frequency.

Firstly, the simulation results of Case 1 are compared with theoretical analysis in Fig. 6. As shown in Fig. 6 (a), each generation unit's frequency response fluctuates around the $\mathrm{CMF}$, and the deviations between them are small, which verifies the effectiveness of studying frequency characteristics based on the CMF. Meanwhile, the validity of FIAPO transfer functions is also verified because the CMF is calculated based on them.

As shown in Fig. 6 (b)-(d), the active power responses $\left(\Delta P_{i}\right)$ obtained by simulations or by the FIAPO transfer functions (taking the simulation frequency as input) almost coincide, which further verifies these FIAPO transfer functions. Besides, the actual power responses $\left(\Delta P_{i}\right)$ fluctuate around the power responses of FIAPO transfer functions when taking the CMF as an input $\left(\Delta P_{i, \mathrm{~cm}}\right)$. This verifies the effectiveness of using $\Delta P_{i, \mathrm{~cm}}$ to study each unit's frequency regulation ability.

Fig. 7 shows the eigenvalues of the original system (in simulation) and the simplified system (CMF) in Case 1. As shown in Fig. 7, although not all eigenvalues of CMF are close to those of the original system, their dominant ones are almost consistent, which further validates the correctness of CMF. The difference may be caused because the voltage dynamics are ignored in CMF.

The CMF is compared with the ASF and COI frequency in Cases 2 and 3. The two cases respectively show the impacts of locations of disturbances or generation units on the CMF. Note that changes of the unit parameters in Cases 3-a and 3-b can be considered as changes of their locations.

As depicted in Fig. 8, there is a large deviation in the bus frequencies between Case $2-\mathrm{a}\left(w_{8}=1.0783, P_{0}=0.3235\right.$ p.u. $), 2$ $\mathrm{b}\left(w_{9}=0.8138, P_{0}=0.2441\right.$ p.u. $)$ or Case 3-a, 3-b. The CMF can reflect these deviations. That is, the obtained trajectory of the CMF is more accurate than the ASF. The COI frequency can also distinguish these cases. However, the COI frequency is the average of the simulation frequencies. Hence, it may not be able to provide much information for theoretical analysis. Furthermore, as shown in the zoomed-in areas of Fig. 8 (b), the COI frequency is not as smooth as the CMF, which demonstrates that inertia may not be the best weighting coefficient for an average frequency when devices are heterogeneous and $w_{i} \neq 1$, as discussed in Section II-C.

In Case 2, especially in Case 2-b, the CMF is not strictly in the center of bus frequencies. This may be due to the fact that the power flow equation involves trigonometric functions and is non-linear. Thus, the linearized analysis will cause certain small errors.

\section{UTFS and frequency indices}

In the following, we adopt the evaluation process proposed in Section IV-B for studying Cases 1, 2, 4, 5, 6 and 7. Here we focus on the validity of the UTFS and indices. In the next subsection, we will further use these cases to compare the regulation capabilities of generation units of different types or with different parameters.

We firstly obtain the weighting vector $\boldsymbol{w}$, the FIAPO transfer functions and the equivalent disturbances. We assume that the power system's requirements on frequency nadir and average RoCoF are that the amplitudes of them cannot exceed $0.5 \mathrm{~Hz}$ or $0.5 \mathrm{~Hz} / \mathrm{s}$ respectively. According to (22), the critical values of indices are $\alpha_{\mathrm{c}}=38.8$ and $J_{\mathrm{zc}}=38.8$ except in Case 2-b, $\alpha_{\mathrm{c}}=29.3$ and $J_{\mathrm{zc}}=29.3$.

Secondly, the UTFS parameters are obtained based on the iterative method proposed in Section III-C, and indices are calculated according to (20) and (21), as shown in TABLE IV.

Lastly, the indices are compared with their critical values to calculate the stability margins. It is concluded that only the frequency nadir in Case 4 does not meet the requirement, where $m_{\text {nadir }}$ is negative.

To verify the above analysis of frequency response characteristics, trajectories of CMF and their approximate damped sinusoids (UTFS) in all these cases are compared in Fig. 9. Moreover, TABLE $\mathrm{V}$ shows the nadir and average RoCoF obtained by the indices, damped sinusoid or CMF. The percentages in the brackets are the relative errors with the CMF as the reference. As shown in Fig. 9 and TABLE V, the 
TABLE IV. UTFS Parameters and Frequency Indices (p.u.).

\begin{tabular}{|c|c|c|c|c|c|}
\hline \multicolumn{2}{|c|}{ Case unit/system } & $J_{\mathrm{u}}, D_{\mathrm{u}}, 1 / K_{\mathrm{u}}$ & $\zeta$ & $\alpha, m_{\text {nadir }}$ & $J_{\mathrm{z}}, m_{\mathrm{ARoCoF}}$ \\
\hline \multirow{4}{*}{1} & SG $(\times 6)$ & $7.13,2.76,1.59$ & - & - & - \\
\hline & GFL $(\times 2)$ & $4.49,20.81,-0.21$ & - & - & - \\
\hline & GFM $(\times 2)$ & $8.00,20.00,0$ & - & - & - \\
\hline & System & $66.88,101.86,8.37$ & 2.04 & $110.8,1.86$ & $111.73,1.88$ \\
\hline \multirow{2}{*}{$2-a$} & $\mathrm{SG}(\times 10)$ & $7.23,2.35,1.86$ & - & - & - \\
\hline & System & $72.34,23.47,18.43$ & 0.32 & $56.68,0.46$ & $83.76,1.16$ \\
\hline \multirow{2}{*}{$2-b$} & $\mathrm{SG}(\times 10)$ & $7.23,2.35,1.86$ & - & - & - \\
\hline & System & $72.34,23.47,18.43$ & 0.32 & $56.68,0.94$ & $83.76,1.86$ \\
\hline \multirow{3}{*}{4} & SG $(\times 6)$ & $7.23,2.35,1.84$ & - & - & - \\
\hline & GFL $(\times 4)$ & $0,0,0$ & - & - & - \\
\hline & System & $42.48,13.79,10.81$ & 0.32 & $33.29,-0.14$ & $49.18,0.27$ \\
\hline \multirow{3}{*}{$5-a$} & SG $(\times 6)$ & $7.21,2.66,1.63$ & - & - & - \\
\hline & GFL $(\times 4)$ & $4.35,20.97,-0.26$ & - & - & - \\
\hline & System & $60.30,102.17,8.48$ & 2.26 & $110.39,1.84$ & $102.29,1.63$ \\
\hline \multirow{3}{*}{$5-b$} & SG $(\times 6)$ & $7.84,0.52,3.70$ & - & - & - \\
\hline & GFL $(\times 4)$ & $-1.14,15.65,3.37$ & - & - & - \\
\hline & System & $41.34,67.63,35.67$ & 0.88 & $96.51,1.87$ & $56.22,0.44$ \\
\hline \multirow{3}{*}{6} & SG $(\times 6)$ & $7.15,2.68,1.62$ & - & - & - \\
\hline & GFM $(\times 4)$ & $8.00,20.00,0$ & - & - & - \\
\hline & System & $74.99,98.31,9.56$ & 1.84 & $111.24,2.01$ & $120.54,2.11$ \\
\hline
\end{tabular}

TABLE V. Comparison of Frequency Nadir and Average RoCoF Obtained by Indices, Damped sinusoid or CMF.

\begin{tabular}{|c|c|c|c|c|c|c|c|}
\hline \multirow[b]{2}{*}{ Case } & \multicolumn{3}{|c|}{ Frequency nadir } & \multicolumn{4}{|c|}{ Average RoCoF } \\
\hline & $\begin{array}{l}\text { Index } \\
(\mathrm{Hz})\end{array}$ & $\begin{array}{l}\text { UTFS } \\
(\mathrm{Hz})\end{array}$ & $\begin{array}{l}\mathrm{CMF} \\
(\mathrm{Hz})\end{array}$ & $\begin{array}{l}\text { Index } \\
(\mathrm{Hz} / \mathrm{s})\end{array}$ & $\begin{array}{l}\text { UTFS } \\
(\mathrm{Hz} / \mathrm{s})\end{array}$ & $\begin{array}{l}\text { CMF } \\
(\mathrm{Hz} / \mathrm{s})\end{array}$ & $\begin{array}{c}t_{\text {naidr }} / 3 \\
(\mathrm{~ms})\end{array}$ \\
\hline 1 & $\begin{array}{l}-0.175 \\
(3.0 \%)\end{array}$ & $\begin{array}{l}-0.170 \\
(0.3 \%)\end{array}$ & -0.170 & $\begin{array}{l}-0.174 \\
(1.9 \%)\end{array}$ & $\begin{array}{l}-0.177 \\
(0.4 \%)\end{array}$ & -0.177 & 691 \\
\hline $2-\mathrm{a}$ & $\begin{array}{l}-0.342 \\
(1.3 \%)\end{array}$ & $\begin{array}{l}-0.348 \\
(0.4 \%)\end{array}$ & -0.347 & $\begin{array}{l}-0.232 \\
(1.9 \%)\end{array}$ & $\begin{array}{l}-0.226 \\
(0.5 \%)\end{array}$ & -0.228 & 867 \\
\hline $2-b$ & $\begin{array}{l}-0.258 \\
(1.3 \%)\end{array}$ & $\begin{array}{l}-0.263 \\
(0.4 \%)\end{array}$ & -0.262 & $\begin{array}{l}-0.175 \\
(1.9 \%)\end{array}$ & $\begin{array}{l}-0.171 \\
(0.5 \%)\end{array}$ & -0.172 & 867 \\
\hline 4 & $\begin{array}{l}-0.583 \\
(1.3 \%)\end{array}$ & $\begin{array}{l}-0.593 \\
(0.4 \%)\end{array}$ & -0.591 & $\begin{array}{l}-0.395 \\
(1.9 \%)\end{array}$ & $\begin{array}{l}-0.386 \\
(0.5 \%)\end{array}$ & -0.387 & 867 \\
\hline $5-\mathrm{a}$ & $\begin{array}{l}-0.176 \\
(3.2 \%)\end{array}$ & $\begin{array}{l}-0.169 \\
(0.5 \%)\end{array}$ & -0.170 & $\begin{array}{l}-0.190 \\
(0.6 \%)\end{array}$ & $\begin{array}{l}-0.195 \\
(2.1 \%)\end{array}$ & -0.191 & 638 \\
\hline $5-b$ & $\begin{array}{l}-0.201 \\
(0.4 \%)\end{array}$ & $\begin{array}{l}-0.202 \\
(0.8 \%)\end{array}$ & -0.200 & $\begin{array}{l}-0.345 \\
(0.7 \%)\end{array}$ & $\begin{array}{l}-0.344 \\
(1.0 \%)\end{array}$ & -0.346 & 373 \\
\hline 6 & $\begin{array}{l}-0.174 \\
(2.8 \%)\end{array}$ & $\begin{array}{l}-0.170 \\
(0.2 \%)\end{array}$ & -0.170 & $\begin{array}{l}-0.161 \\
(2.9 \%)\end{array}$ & $\begin{array}{l}-0.165 \\
(0.7 \%)\end{array}$ & -0.165 & 733 \\
\hline
\end{tabular}

approximate trajectories are very close to the CMF for a period, and the relative errors of frequency factors are all less than 5\%, which verifies the effectiveness of the UTFS and indices.

To demonstrate the convergence performance of the iterative method, Fig. 9 (a) shows the time-domain response of CMF and the approximate damped sinusoid (based on UTFS) of each iteration in Case 1. The iteration initial UTFS parameters of all devices are identically chosen as $J_{\mathrm{u}}=8, D_{\mathrm{u}}=0$ and $1 / K_{\mathrm{u}}=0$. As shown in Fig. 9 (a), the damped sinusoid is close to the CMF after the first iteration, and converges after the third time. The calculation time of each iteration is about $75 \mathrm{~ms}$.

It is also noteworthy that in Case 1, the initial RoCoF calculated by the total inertia (including virtual inertia) is $-0.243 \mathrm{~Hz} / \mathrm{s}$ (which is the situation of iteration 0 in Fig. 9 (a)).

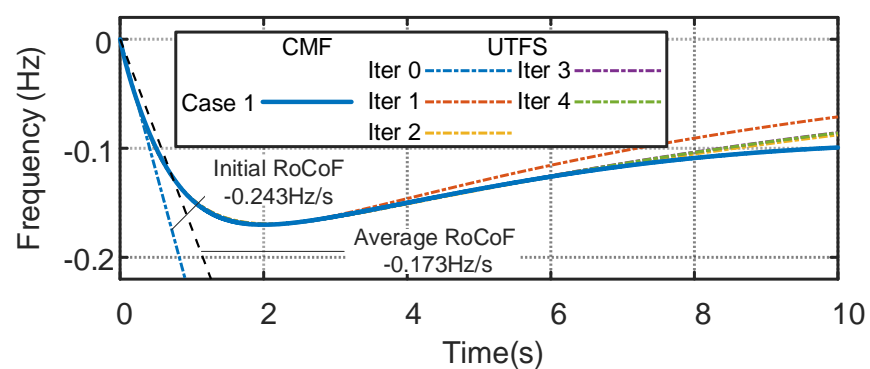

(a)

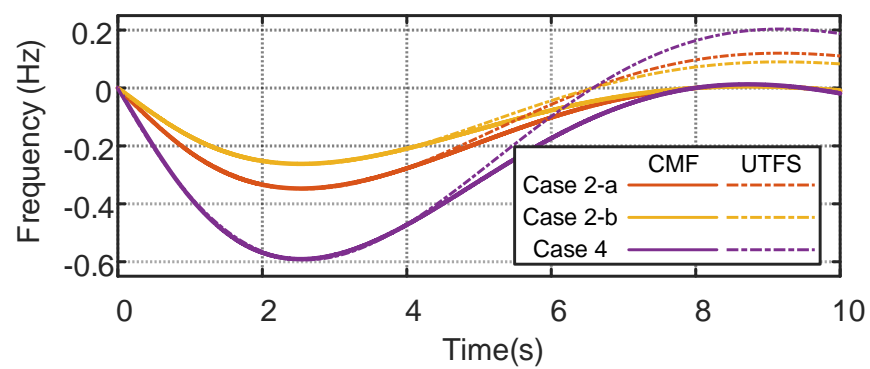

(b)

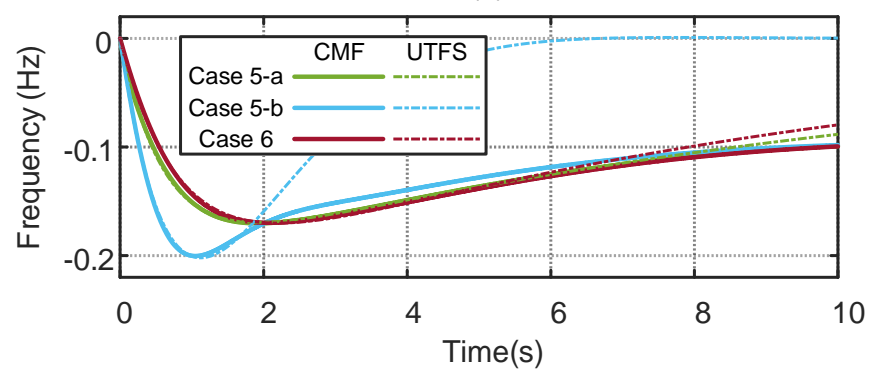

(c)

Fig. 9. Comparison of the CMF trajectories and their approximations based on the UTFS in Case 1 (a), Case 2-a, 2-b, 4 (b) and Case 5-a, 5-b, 6 (c).

However, the initial $\mathrm{RoCoF}$ is $40 \%$ smaller than the average RoCoF $-0.173 \mathrm{~Hz} / \mathrm{s}$ calculated by the proposed FDSC. As shown in the figure, the average RoCoF can represent the frequency change trend better than the initial RoCoF, which indicates that FDSC is a more representative index than inertia.

Although the UTFS parameters of the generation units are recalculated in every case, they will not change significantly as long as the unit itself remains the same and the system CMF does not change significantly, see for instance, the UTFS parameters of SG in all cases in TABLE IV (except in Case 5$\mathrm{b}$ where the system frequency is much different from others). Therefore, based on the UTFS parameters, the system frequency response characteristics can be predicted when the system parameters change. We take Case 4 as an example. Consider that the virtual inertia and damping coefficient of converters are respectively increased to 8 and 20 (to simplify the analysis, the converter is replaced by a GFM converter, which is in fact the situation of Case 6). If we directly add [ $\Delta J_{\mathrm{us}}$, $\left.\Delta D_{\text {us }}\right]=\left(w_{2}+w_{4}+w_{6}+w_{8}\right) \times[8,20]=[33.02,82.56]$ to the UTFS parameters of Case 4, the system UTFS parameters and frequency indices are respectively $J_{\text {us }}=75.50, D_{\text {us }}=96.34$, $1 / K_{\text {us }}=10.82$, and $\alpha=111.36, \quad J_{z}=118.80$. These UTFS parameters and indices are very close to those of Case 6 shown in TABLE IV, which verifies the effectiveness of the above parametric variation analysis. 
As shown in TABLE IV, the largest damping ratio in these cases is 2.26 (Case 5-a). It is smaller than 2.5, which meets the conditions of Proposition 1. We do not analyze Case 3 because its damping ratio is infinite $\left(1 / K_{\mathrm{us}}=0\right)$, which will be our future research.

\section{Frequency regulation capability evaluation}

In Case 1, all the generation units have the same inertia or virtual inertia. However, the GFL converter's power response when taking the CMF as input is slower than the SG or GFM converter right after disturbance (0 0.2s), as depicted in Fig. 6 (b)-(d). Moreover, although all the units have the same PFC coefficient, the power output of the SG is much less than the converters during transients (e.g., 1s-3s). These illustrate that the frequency regulation capabilities of the devices cannot be directly quantified by their control parameters, even though these parameters have analogous physical meanings. This problem can be solved by using the units' UTFS parameters. As shown in TABLE IV, $J_{\mathrm{u}}$ of the GFL converter is smaller than the SG and GFM converter in Case 1. Besides, $D_{\mathrm{u}}$ of SG is much smaller than the two converters. These results are consistent with the phenomenon observed in Fig. 6.

The frequency regulation abilities of SGs and converters can be compared in Cases 1, 2-a and 4. As depicted in Fig. 9 (a), (b), the amplitude of the frequency nadir and average RoCoF in Case $4\left(J_{\mathrm{GFL}}=D_{\mathrm{GFL}}=0\right)$ are much larger than those in Case 2a, which demonstrates that replacing SGs with converters that do not participate in frequency regulation will deteriorate the power system's frequency stability. However, these frequency factors in Case 1 are even better than in Case 2-a, which reveals the potential of the converters in frequency regulation.

Furthermore, the capabilities of GFL and GFM converters are compared in Case 5 and 6, where converters have the same virtual inertial and PFC coefficient. As shown in Fig. 9 (c), the overall frequency trajectory in Case $5-\mathrm{a}\left(T_{\mathrm{D}}=0.01 \mathrm{~s}\right)$ is roughly the same as that in Case 6. However, in Case 5-b $\left(T_{\mathrm{D}}=0.5 \mathrm{~s}\right)$, the frequency response is much worse. It illustrates that a longer delay will weaken the device's frequency regulation capability, which is in consistency with intuition and can be quantified by the UTFS parameters. Compared with Case 5-a, the $J_{\mathrm{u}}$ and $D_{\mathrm{u}}$ of the GFL converter in Case 5-b are much smaller, and only $1 / K_{\mathrm{u}}$ increases slightly.

\section{DISCUSSIONS}

Our study is mainly based on three assumptions, i.e., 1) constant device voltage amplitude, 2) the system is linear, and 3 ) the system model is known. Assumption 1 is reasonable in power systems where generation units can regulate voltage fast. Some nonlinear dynamics have negligible impacts on the frequency response, such as the dead zone of the governor, and thus can be ignored.

There might be cases where the voltage dynamics and nonlinearity have a visible impact. Here we discuss the possible solutions to relax these assumptions.

1) If the voltage dynamics are not negligible, the devices need to be represented by the transfer function matrices with voltage, phase angle (frequency) as inputs and active, reactive power as outputs. Accordingly, the interface of the power flow equation needs to be modified. How to obtain the global frequency in such a situation is an ongoing study. We note that one possible direction is to take advantage of the rotational invariance of reactive power flow to phase angle, considering that voltage dynamics are close-coupled with reactive power.

2) It is in general difficult to deal with a large-scale nonlinear system and express the system global frequency analytically. To this end, the following practical method may be adopted. Note that the model simplification (Section III) and the subsequent frequency evaluation (Section IV) do not need the linear condition. Therefore, we only analyze how to deal with nonlinearity in the calculation of CMF (Section II). There are two aspects of the nonlinearity that affect the CMF, i.e., the nonlinearity of the generation units and the power flow. For units with nonnegligible nonlinearity, we can replace their FIAPO transfer functions with the nonlinear models (represented by block diagram or differential-algebraic equation) in the calculation of the CMF, while the weighting coefficients remain the same. When the nonlinearity of power flow cannot be ignored, one may use the weighting vector recalculated according to the post-disturbance power flow to improve the accuracy, as the power flow affects CMF only via the weighting vector. It is worth mentioning that the analysis will be more complicated when the above methods are used. For example, the weighting vector will be a function of the disturbance if it is based on the post-disturbance power flow.

3) As for the third assumption, if the system model is unknown, one can try to identify the system model, e.g., identifying the networks [27] and the devices [28]. Note that our model-based method aims to provide theoretical foundations for the system analysis, and future work can include, for instance, the fast calculations, effective identification of the devices, and real-world implementations.

\section{CONCLUSION}

This paper defines the common-mode frequency (CMF) for inverter-penetrated power systems, which is extracted from the frequencies of all the generation units. We rigorously showed that the CMF well represents the global frequency in the system level. Moreover, the CMF links the device-level frequency characteristics to the system-level frequency characteristics, which can be used to study how the design of devices affects the system's frequency responses. Based on the CMF, we presented an analytical method for assessing the frequency response characteristics of inverter-penetrated power systems. It has been demonstrated that:

1) The CMF is superior to the conventional ASF. The proposed CMF is more theoretically rigorous and more accurate. The expression of the CMF demonstrates that the generation units and the disturbances have different weightings on the global frequency according to their locations, which cannot be inferred from the ASF. Moreover, similar to the COI frequency, the $\mathrm{CMF}$ can also be obtained by weighting the bus frequencies. However, the weighting vector of CMF is $\boldsymbol{v}_{1}(s)$, which is shown to be a better choice than the inertia vector of the $\mathrm{COI}$ frequency.

2) To simplify the analysis of the devices' regulation 
capabilities and the system frequency characteristics, we propose a UTFS to approximate the FIAPO transfer functions of heterogeneous devices (SGs, IBGs, etc.). In this way, the distinct regulation characteristics of different devices can be captured by three key parameters in a unified transfer function, which facilitate the comparison between different devices and the calculation of the CMF.

3) The proposed indices, i.e., FDDC and FDSC, can quantify the frequency nadir and average RoCoF accurately. These two indices help to intuitively understand the influences of devicelevel frequency regulations on the system frequency response.

Our analyses focus on the global frequency, i.e., CMF, which is a decomposed frequency component related to the eigenvalue 0 of the reduced Laplacian matrix $\boldsymbol{L}_{\mathrm{r}}$. In fact, the other frequency components corresponding to the other eigenvalues represent the frequency oscillations in power systems [8]-[9], which can be called the difference-mode frequencies (DMFs). We will consider the analyses of DMFs as part of our future work.

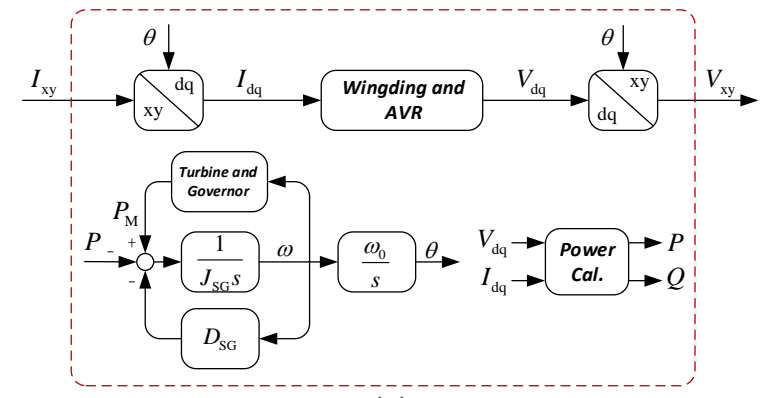

(a)

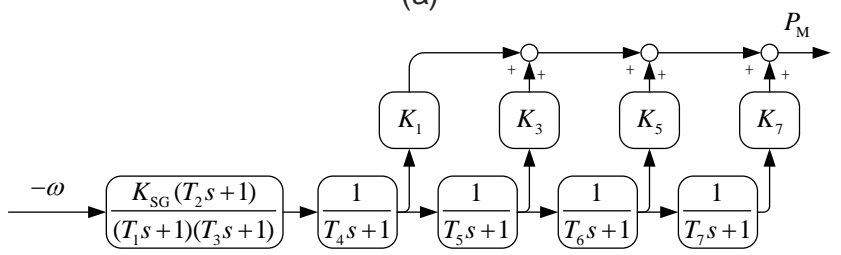

(b)

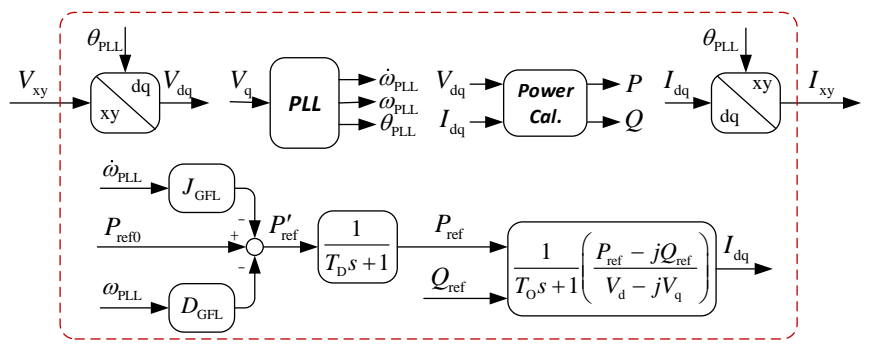

(c)

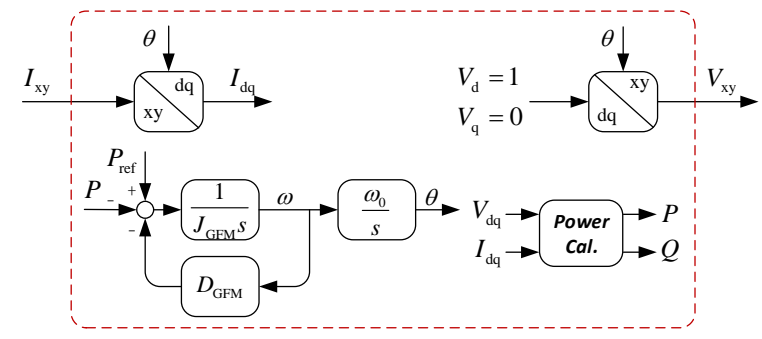

(d)

Fig. 10. Model of SG (a), its turbine-governor (b), GFL converter (c) and GFM converter (d).

\section{APPENDIX}

\section{A. Models and FIAPO transfer functions of generation units}

a) SG: The model of SG and its turbine-governor [25]-[26] are respectively depicted in Fig. 10 (a), (b). The winding, the AVR of the SG, and the power calculation are formulated as follows

$$
\begin{aligned}
& \text { (Winding) }\left\{\begin{aligned}
T_{\mathrm{d}}^{\prime} s E_{\mathrm{q}}^{\prime} & =E_{\mathrm{fd}}-E_{\mathrm{q}}^{\prime}-\left(X_{\mathrm{d}}-X_{\mathrm{d}}^{\prime}\right) I_{\mathrm{d}} \\
V_{\mathrm{d}} & =X_{\mathrm{q}} I_{\mathrm{q}} \\
V_{\mathrm{q}} & =E_{\mathrm{q}}^{\prime}-X_{\mathrm{d}} I_{\mathrm{d}}
\end{aligned}\right. \\
& (\mathrm{AVR}) \quad\left\{\begin{array}{l}
E_{\mathrm{fd}}=\frac{K_{\mathrm{AVR}}}{T_{\mathrm{AVR}} s+1}\left(V_{\mathrm{ref}}-|V|\right) \\
|V|=\sqrt{V_{\mathrm{d}}^{2}+V_{\mathrm{q}}^{2}}
\end{array}\right. \\
& \text { (Power Cal.) }\left\{\begin{array}{l}
P=V_{\mathrm{d}} I_{\mathrm{d}}+V_{\mathrm{q}} I_{\mathrm{q}} \\
Q=-V_{\mathrm{d}} I_{\mathrm{q}}+V_{\mathrm{q}} I_{\mathrm{d}}
\end{array}\right.
\end{aligned}
$$

where $J_{\mathrm{SG}}$ and $D_{\mathrm{SG}}$ are inertia and damping coefficient respectively; $\omega$ and $\theta$ are rotor speed and rotor angle respectively; $T_{\mathrm{d}}^{\prime}$ and $X_{\mathrm{d}}^{\prime}$ are $\mathrm{d}$-axis transient time constant and transient internal reactance; $E_{\mathrm{q}}^{\prime}$ and is q-axis transient internal voltage; $I_{\mathrm{d}}$ and $I_{\mathrm{q}}$ are d-axis and q-axis stator currents; $V_{\mathrm{d}}$ and $V_{\mathrm{q}}$ are $\mathrm{d}$-axis and q-axis stator voltage; $E_{\mathrm{fd}}$ is the excitation voltage; $K_{\mathrm{AVR}}$ and $T_{\mathrm{AVR}}$ are the gain and time constant of AVR; $|V|$ and $V_{\text {ref }}$ are voltage amplitude and its reference value respectively; $P$ and $Q$ are active and reactive power respectively; $P_{\mathrm{M}}$ is the mechanical power; $K_{\mathrm{SG}}, K_{1}, K_{3}, K_{5}$ and $K_{7}$ are gains of the turbine governor, and $T_{1}-T_{7}$ are its time constants.

Note that the above model is interfaced with the transmission network by voltage and current. It is the common practice for simulations in Matlab/Simulink. However, theoretical analysis of frequency will be easier if the interface is active power and frequency. To this end, we formulate the FIAPO transfer function based on the above model as follows.

The input frequency of the SG's FIAPO transfer function is its internal frequency, i.e., rotor speed. This is because the change of internal frequency is the reason why the SG's output active power changes (released rotor kinetic energy and response of turbine-governor). Consequently, the internal impedance needs to be regarded as a part of the network. In fact, the internal impedance can also be ignored in the network part, which is explained when we discuss the device aggregation at the end of Section II-C.

By transforming from the swing equation, the FIAPO transfer function of the SG can be formulated as

$$
G_{\mathrm{SG}}(s)=J_{\mathrm{SG}} s+D_{\mathrm{SG}}+G_{\mathrm{T}}(s)
$$

where $G_{\mathrm{T}}(s)$ is the transfer function of the turbine-governor, as illustrated in Fig. 10 (b).

b) GFL converter: The GFL converter model in [20] is adopted with slight modification, as shown in Fig. 10 (c). The $\omega_{\text {PLL }}, \dot{\omega}_{\text {PLL }}$ and $\theta_{\text {PLL }}$ are respectively the frequency, its derivate and phase angle estimated by the phase-locked loop (PLL), which is formulated as (26); $P_{\text {refo }}$ is the set point of active power; $K_{\mathrm{P}}$ and $K_{\mathrm{I}}$ are the PI parameters of PLL; $J_{\mathrm{GFoll}}$ and $D_{\mathrm{GFoll}}$ are virtual inertia and droop coefficient; $T_{\mathrm{D}}$ is the time 
constant of a low-pass filter which is used to investigate the impact of the time delay on frequency regulation effect; $T \mathrm{o}$ is the time constant of the power output delay.

$$
\left\{\begin{aligned}
V_{\mathrm{q}} & =|V| \sin \left(\theta-\theta_{\mathrm{PLL}}\right)^{\prime} \quad \dot{\theta}=\omega_{0} \omega \\
\dot{\theta}_{\mathrm{PLL}} & =\omega_{0} \omega_{\mathrm{PLL}} \\
T_{\mathrm{F}} \dot{\omega}_{\mathrm{PLL}} & =-\omega_{\mathrm{PLL}}+K_{\mathrm{P}} V_{\mathrm{q}}+K_{\mathrm{I}} \int V_{\mathrm{q}}
\end{aligned}\right.
$$

where $\omega$ and $\theta$ are frequency and phase angle of the terminal voltage; $T_{\mathrm{F}}$ is the time constant of a filter, based on which the frequency derivate can be estimated [20].

It can be deduced that the change of the terminal frequency $\omega$ will cause the converter output power change. Thus, the terminal frequency is considered as the interface frequency of the generation and network. According to Fig. 10 (c), (26), and assuming $|V|=1$, the FIAPO transfer function (between $\Delta \omega$ and $\left.\Delta P_{\mathrm{O}}\right)$ is as follows

$$
\underbrace{\frac{G_{\mathrm{GFL}}(s)=}{T_{\mathrm{F}} s^{3}+s^{2}+s K_{\mathrm{P}} \omega_{0}+K_{\mathrm{I}} \omega_{0}}}_{\text {PLL and vitrual inertia }} \times \underbrace{\frac{1}{T_{\mathrm{D}} s+1}}_{\text {extra delay }} \times \underbrace{\frac{1}{T_{0} s+1}}_{\text {power output }}
$$

c) GFM converter: The model of the GFM converter used in this paper is exactly the same as in [20], shown in Fig. 10 (d), where $J_{\mathrm{GFM}}$ and $D_{\mathrm{GFM}}$ are the virtual inertia and damping coefficient respectively. It is a virtual synchronous machine with constant internal voltage amplitude and zero internal impedance. Similar to the SG, the FIAPO transfer function of the GFM converter is:

$$
G_{\mathrm{GFM}}(s)=J_{\mathrm{GFM}} s+D_{\mathrm{GFM}}
$$

\section{B. Reduction of passive buses}

Firstly, if a bus contains both constant power loads and other loads, the constant power loads are considered as located at a slack bus that is connected to the original bus with a small impedance. Then, the internal buses, constant impedance and constant current load buses can be eliminated by the Kron reduction [22], resulting in a network admittance matrix $\boldsymbol{Y}$ which only contains constant power load buses and generation buses.

After that, we write the linearized active power flow equation involving the two remaining kinds of buses:

$$
\left[\begin{array}{l}
\Delta \boldsymbol{P}_{\mathrm{G}}(s) \\
\Delta \boldsymbol{P}_{\mathrm{P}}(s)
\end{array}\right]=\left[\begin{array}{ll}
\boldsymbol{L}_{11} & \boldsymbol{L}_{12} \\
\boldsymbol{L}_{21} & \boldsymbol{L}_{22}
\end{array}\right]\left[\begin{array}{l}
\Delta \boldsymbol{\theta}_{\mathrm{G}}(s) \\
\Delta \boldsymbol{\theta}_{\mathrm{P}}(s)
\end{array}\right]
$$

where subscripts $\mathrm{G}$ and $\mathrm{P}$ denote generation units and constant power loads, respectively; $\Delta \boldsymbol{P}_{\mathrm{G}}, \Delta \boldsymbol{P}_{\mathrm{P}}$ are vectors of active power injected into the network; $\Delta \boldsymbol{\theta}_{\mathrm{G}}, \Delta \boldsymbol{\theta}_{\mathrm{P}}$ are vectors of phase angles; $\boldsymbol{L}_{11}, \boldsymbol{L}_{12}, \boldsymbol{L}_{21}$ and $\boldsymbol{L}_{22}$ are four appropriate submatrices of the Laplacian matrix $\boldsymbol{L}$, whose elements are given as

$$
\left\{\begin{array}{l}
\boldsymbol{L}[i, i]=\left|V_{i}\right| \sum_{j=1, j \neq i}^{n+m}\left|V_{j}\right|\left(-G_{i j} \sin \theta_{i j 0}+B_{i j} \cos \theta_{i j 0}\right) \\
\boldsymbol{L}[i, j]=-\left|V_{i} \| V_{j}\right|\left(-G_{i j} \sin \theta_{i j 0}+B_{i j} \cos \theta_{i j 0}\right)
\end{array}\right.
$$

where $m$ is the number of constant power load buses; $\left|V_{i}\right|$ is the steady voltage amplitude at bus $i ; \theta_{i j 0}$ is the steady angle difference between bus $i$ and bus $j$; $G_{i j}+\mathrm{j} B_{i j}$ is element of $\boldsymbol{Y}=$
$\boldsymbol{G}_{\mathrm{B}}+\mathrm{j} \boldsymbol{B}_{\mathrm{B}}$, the subscript B is used to distinguish $\boldsymbol{G}_{\mathrm{B}}$ from $\boldsymbol{G}(s)$.

For constant power loads, we have $\Delta \boldsymbol{P}_{\mathrm{P}}=\mathbf{0}$. Substituting it into (29) gives

$$
\Delta \boldsymbol{P}_{\mathrm{G}}(s)=\boldsymbol{L}_{\mathrm{r}} \Delta \boldsymbol{\theta}_{\mathrm{G}}(s), \quad \boldsymbol{L}_{\mathrm{r}}:=\boldsymbol{L}_{11}-\boldsymbol{L}_{12} \boldsymbol{L}_{22}^{-1} \boldsymbol{L}_{21}
$$

where $\boldsymbol{L}_{\mathrm{r}}$ is the reduced Laplacian matrix. Note that the constant power load buses are eliminated.

Considering that $\Delta \boldsymbol{\theta}=\omega_{0} \mathbf{s}^{-1} \Delta \boldsymbol{\omega}$, (31) can be rewritten as (2), where the subscript $\mathrm{G}$ is omitted for notation simplicity.

It is worth mentioning that the sum of each row in the matrix $\boldsymbol{L}$ is 0 . Hence, $\boldsymbol{L}$ has a zero eigenvalue, and the corresponding right eigenvector is $\mathbf{1}$ [8]. It is easy to prove that $\boldsymbol{L}_{\mathrm{r}}$ in (31) has the same property, i.e., it has eigenvalue 0 and right eigenvector 1 [22]. This property of $\boldsymbol{L}_{\mathrm{r}}$ is important and will be used in the derivation of the CMF.

\section{Proof of Proposition 1}

a) The time-domain expression of $\Delta \omega_{\mathrm{cm}}^{\prime}$ in (16) is

$$
\Delta \omega_{\text {cm }}^{\prime}(t)=-\frac{P_{0}}{J_{\text {us }} \omega_{\mathrm{d}}} \mathrm{e}^{\sigma t} \sin \left(\omega_{\mathrm{d}} t\right)
$$

Note that when $\omega_{\mathrm{d}}=\omega_{\mathrm{n}} \sqrt{1-\zeta^{2}}$ equals to $0(\zeta=1)$ or is an imaginary number $(\zeta>1)$, the expression in (32) is still applicable. When $\zeta=1, \Delta \omega^{\prime} \mathrm{cm}(t)$ can be defined by limit. And when $\zeta>1$, the complex trigonometric function $\sin (i x)=$ $i \sinh (x)$ can be substituted into (32) to calculate $\Delta \omega_{\mathrm{cm}}^{\prime}$.

Take the derivative of $\Delta \omega_{\mathrm{cm}}^{\prime}$ with respect to $t$. The first time when the derivative equals 0 is $t_{\text {nadir }}$. And then the expression of $\Delta \omega_{\text {nadir }}$ can be obtained, as shown in (17).

Substituting the expressions of $\zeta, \omega_{\mathrm{n}}, \omega_{\mathrm{d}}$ and $\sigma$ into (17)-(18) , then $\Delta \omega_{\text {nadir }}$ and $\Delta \omega^{\prime}{ }_{\text {nadir }}$ can be expressed as

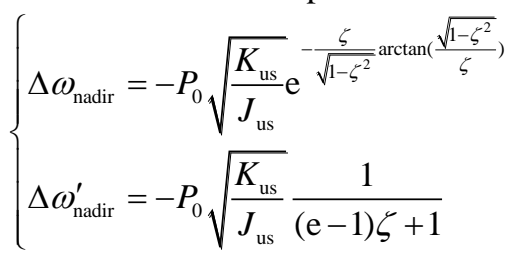

According to (33), the relative error between $\Delta \omega_{\text {nadir }}$ and $\Delta \omega_{\text {nadir }}^{\prime}$ only depends on $\zeta$. As shown in Fig. 11, when $0 \leq \zeta \leq 2.5$, the relative error is less than $5 \%$.

b) Similar to the above discussion, the average RoCoF $\Delta \dot{\omega}_{01 t_{1}}$ and its approximation $\Delta \dot{\omega}_{01}^{\prime} t_{1}$ can be expressed as

$$
\left\{\begin{array}{l}
\Delta \dot{\omega}_{01} t_{1}=-\frac{P_{0}}{J_{\text {us }}} \frac{\mathrm{e}^{\frac{1}{n_{t}} \arctan \left(\frac{\sqrt{1-\zeta^{2}}}{\zeta}\right)} \cdot \frac{\zeta}{\sqrt{1-\zeta^{2}}} \sin \left(\frac{1}{n_{t}} \arctan \left(\frac{\sqrt{1-\zeta^{2}}}{\zeta}\right)\right)}{\frac{1}{n_{t}} \arctan \left(\frac{\sqrt{1-\zeta^{2}}}{\zeta}\right)} \\
\Delta \dot{\omega}_{01}^{\prime} t_{1}=-\frac{P_{0}}{J_{\text {us }}} \frac{1}{[(\mathrm{e}-1) \zeta+1]^{\frac{1}{n_{t}}}}
\end{array}\right.
$$

When $n_{\mathrm{t}}$ is determinate, the relative error between them also only depends on $\zeta$. Fig. 11 shows the relative error when $n_{\mathrm{t}}=3$. And it is less than $5 \%$ when $0 \leq \zeta \leq 2.5$. 


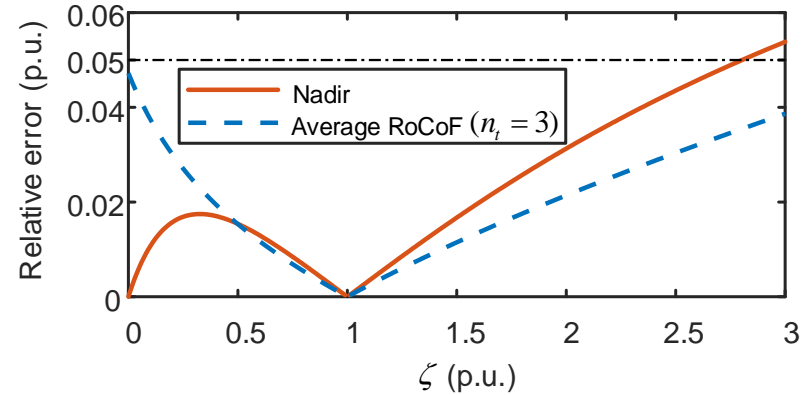

Fig. 11. Relative errors of the frequency nadir and average RoCoF obtained by indices.

\section{REFERENCES}

[1] F. Milano, F. Dörfler, G. Hug, D. Hill, and G. Verbic. "Foundations and challenges of low-inertia systems," in Proc. Power Syst. Comput. Conf. (PSCC), Jun. 2018.

[2] A. Tayyebi, D. Groß, A. Anta, F. Kupzog, and F. Dörfler. "Frequency stability of synchronous machines and grid-forming power converters," IEEE Trans. Emerg. Sel. Topics Power Electron., vol. 8, no. 2, pp. 1004 $1018,2020$.

[3] National grid ESO, "Technical Report on the events of 9 August 2019," 2019.

[4] B. Magness, "Review of Feb. 2021 Extreme Cold Weather Event-Ercot Presentation," 2021.

[5] Y. Wen, W. Li, G. Huang, and X. Liu. "Frequency dynamics constrained unit commitment with battery energy storage," IEEE Trans. Power Syst., vol. 31. no. 6 pp. 5115-5125, 2016.

[6] L. Hao, Y. Qiao, Z. Lu, B. Zhang, and F. Teng. "Frequency constrained stochastic planning towards a high renewable target considering frequency response support from wind power," IEEE Trans. Power Syst., vol. 36, no. 5, pp. 4632-4644, 2021.

[7] M. L. Chan, R. D. Dunlop, and F. Schweppe, "Dynamic equivalents for average system frequency behaviour following major disturbances," IEEE Trans. Power App. Syst., vol. PAS-91, no. 4, pp. 1637-1642, 1972.

[8] F. Paganini and E. Mallada. "Global analysis of synchronization performance for power systems: bridging the theory-practice gap," IEEE Trans. Automat. Contr., vol. 65, no. 7, pp. 3007-3022, 2019.

[9] H. Gao, H. Yuan, H. Xin, L. Huang, C. Feng. "Nodal frequency performance of power networks," in International Conference on Advanced Power System Automation and Protection (APAP), 2019.

[10] H. Gao, H. Xin, L, Huang, et al. "Characteristic analysis and quantification of common mode frequency in power systems with high penetration of renewable resources," Proceedings of the CSEE, vol. 41, no. 3, pp. 890-900, 2021.

[11] U. Rudez and R. Mihalic, "Analysis of underfrequency load shedding using a frequency gradient," IEEE Trans. Power Deliv., vol. 26, no. 2, pp. 565-575, Apr. 2011.
[12] N. Maruejouls, T. Margotin, M. Trotignon, P. L. Dupuis, and J.-M Tesseron, "Measurement of the load frequency control system service: Comparison between American and European indicators," IEEE Trans. Power Syst., vol. 15, pp. 1382-1387, 2000.

[13] Y. Bian, H. Wyman-Pain, F. Li, R. Bhakar, S. Mishra, and N. P. Padhy, "Demand side contributions for system inertia in the GB power system," IEEE Trans. Power Syst., vol. PP, no. 99, pp. 1-1, 2017.

[14] P. M. Anderson and M. Mirheydar, "A low-order system frequency response model," IEEE Trans. Power Syst., vol. 5, no. 3, pp. 720-729, Aug. 1990.

[15] L. Sigrist, I. Egido, and L. Rouco, "Principles of a centralized UFLS scheme for small isolated power systems," IEEE Trans. Power Syst., vol. 28, no. 2, pp. 1779-1786, May 2013.

[16] S. Guggilam, C. Zhao, E. Dall'Anese, Y. C. Chen, and S. Dhople, "Optimizing DER participation in inertial and primary-frequency response," IEEE Trans. Power Syst., vol. 33, no. 5, pp. 5194-5205, 2018.

[17] Liu, L., W. Li, J. Shen, C. Jin, and K. Wen. "An analytical model for frequency nadir prediction following a major disturbance," IEEE Trans. Power Syst. vol. 35, no. 4, pp. 2527-2536, 2020.

[18] P. Ju, Y. Zheng, Y. Jin, C. Qin, Y. Jiang, and L. Cao. "Analytic assessment of the power system frequency security," IET Gener. Transm. Distrib., 2021.

[19] E. Rakhshani, D. Gusain, V. Sewdien, J. L. Rueda Torres, and M. A. M. M. Van Der Meijden, "A key performance indicator to assess the frequency stability of wind generation dominated power system," IEEE Access, vol. 7, pp. $130957-130$ 969, 2019.

[20] B. K. Poolla, D. Groß, and F. Dörfler, "Placement and implementation of grid-forming and grid-following virtual inertia and fast frequency response," IEEE Trans. Power Syst., vol. 34, no. 4, pp. 3035-3046, 2019.

[21] C. Yang, L. Huang, H. Xin, and P. Ju, "Placing grid-forming converters to enhance small signal stability of PLL-integrated power systems," IEEE Trans. Power Syst., vol. 36, no. 4, pp. 3563-3573, Jul. 2021.

[22] F. Dörfler and F. Bullo, "Kron reduction of graphs with applications to electrical networks." IEEE Trans. Circuits Syst., vol. 60, no. 1, pp. 150163, 2013.

[23] F. Milano and Á. Ortega, "Frequency divider," IEEE Trans. Power Syst., vol. 32, no. 2, pp. 1493-1501, Mar. 2017.

[24] I. Hiskens, "39-bus system (New England Reduced Model)," IEEE PES Task Force on Benchmark Systems for Stability Controls, Tech. Rep., Nov. 2013.

[25] P. Kundur, Power System Stability and Control. New York: McGrawHill, 1994.

[26] IEEE Committee. "Dynamic models for steam and hydro turbines in power system studies," IEEE Trans. Power App. Syst., vol. PAS-92, no. 6, pp.1904-1915, 1973.

[27] L. Huang, H. Xin, Z. Li, P. Ju, H. Yuan, and G. Wang. "Identification of generalized short-circuit ratio for on-line stability monitoring of wind farms," IEEE Trans. Power App. Syst., vol. 35, no. 4, pp. 3282-3285, 2020.

[28] F. Milano and Á. Ortega, "A method for evaluating frequency regulation in an electrical grid - Part I: Theory," IEEE Trans. Power Syst., vol. 36, no. 1, pp. 183-193, Jan. 2021. 